\title{
Sacraoxides A-G, Bioactive Cembranoids from Gum Resin of Boswellia sacra
}

\author{
Bingyang Zhang ${ }^{1+}$, Di Liu ${ }^{1+}$, Wenyue $\mathrm{Ji}^{1}$, Kouharu Otsuki ${ }^{2}$, Koji Higai ${ }^{2}$, Feng Zhao ${ }^{3 *}$, Wei Li ${ }^{2 *}$, \\ Kazuo Koike ${ }^{2}$ and Feng Qiu ${ }^{1 *}$ \\ ${ }^{1}$ School of Chinese Materia Medica, Tianjin University of Traditional Chinese Medicine, Tianjin, China, ${ }^{2}$ Faculty of Pharmaceutical \\ Sciences, Toho University, Tokyo, Japan, ${ }^{3}$ School of Pharmacy, Key Laboratory of Molecular Pharmacology and Drug Evaluation \\ (Yantai University), Ministry of Education, Collaborative Innovation Center of Advanced Drug Delivery System and Biotech Drugs in \\ Universities of Shandong, Yantai University, Yantai, China
}

Seven undescribed cembranoids, sacraoxides $A-G(\mathbf{1}, \mathbf{3}-\mathbf{8})$ were isolated from the gum resin of Boswellia sacra. Their structures were elucidated by extensive physicochemical and spectroscopic analysis, as well as ECD calculation, modified Mosher's method and $\mathrm{X}$-ray diffraction crystallography. Compounds $\mathbf{6}$ and $\mathbf{7}$ exhibited inhibitory activities on nitric oxide (NO) production induced by lipopolysaccharide in RAW264.7 cells with $I_{50}$ values of $24.9 \pm 1.7$ and $36.4 \pm 2.9 \mu \mathrm{M}$.

Keywords: Boswellia sacra, olibanum, phytochemistry, cembranoids, structure elucidation, anti-inflammatory

\section{INTRODUCTION}

Cembranoids are a class of diterpenes biosynthesized from the cyclization of geranylgeranyl diphosphate to generate a 14-membered ring backbone decorated with a variety of oxidation patterns (Li and Pattenden, 2011). Natural occurrence of cembranoids has been found from both terrestrial and marine organisms, which showed not only a large structural diversity but also a wide range of biological activities (Yang et al., 2012). Plant-derived cembranoids have a relatively limited distribution, and have been reported mainly from tobacco (Yan et al., 2016), as well as the genera of Croton, Euphorbia, Macaranga (Euphorbiaceae), Pinus (Pinaceae), Echinodorus (Alismataceae), and Boswellia (Burseraceae), etc (Wahlberg et al., 1992; Yang et al., 2012).

Olibanum is an aromatic oleogum resin that exudes from incisions in the bark of Boswellia trees and has been used as incense and perfumes since antiquity. Olibanum has also been used in traditional medicines for the purpose of relieving pain and removing blood stasis. Cembranoids and triterpenes were reported as the bioactive constituents responsible for these effects (Banno et al., 2006; Takada et al., 2006; Al-Harrasi et al., 2019). Boswellia sacra, known as Olibanum-tree or Frankincense, is a small deciduous tree that is native to the Arabian Peninsula and northeastern Africa, and is one of the plants known to produce olibanum. Previous chemical investigations on the gum resin from $B$. sacra have reported the isolation of a number of cembranoids with neuroprotective, hepatoprotective, anti-inflammatory and anti-depression activities (Moussaieff et al., 2012; Pollastro et al., 2016; Wang et al., 2020).

As part of a continuing research for the discovery of bioactive natural products from medicinal plants (Xia et al., 2017; Zhang et al., 2020), a chemical investigation was carried out on the gum resin of $B$. sacra. Herein, we report the isolation and structural elucidation of nine cembranoids (1-9) as well as their inhibitory activities against lipopolysaccharide (LPS)-induced NO production in RAW 264.7 mouse monocyte-macrophages. 


\section{MATERIAL AND METHODS}

\section{General Experimental Procedures}

Optical rotations were measured on a JASCO P-2200 polarimeter (JASCO Corp., Tokyo, Japan) in a $0.5 \mathrm{dm}$ cell. The UV spectra were obtained with a Shimadzu UV 2201 spectrophotometer (Shimadzu Corp., Tokyo, Japan). The ECD spectra were measured on a JASCO J-1500 spectropolarimeter (JASCO Corp., Tokyo, Japan) in a $10 \mathrm{~mm}$ cell. The ${ }^{1} \mathrm{H}$ and ${ }^{13} \mathrm{C}$ NMR spectra were performed on Bruker AV-600 spectrometer (Bruker BioSpin, Zurich, Switzerland) or a JEOL ECA-500 spectrometer (JEOL Corp., Tokyo, Japan) with the measuring deuterated solvent as the internal reference. The chemical shifts are expressed in $\delta(\mathrm{ppm})$ and reported as s (singlet), $\mathrm{d}$ (doublet), $\mathrm{t}$ (triplet), dd (doublet of doublets), dt (doublet of triplets), ddd (doublet of doublet of doublets), ddt (doublet of doublet of triplets), hept (heptet), br (broad) and $\mathrm{m}$ (multiplet), respectively. HRESIMS was conducted using a Waters (Milford, MA) ACQUITY SYNAPTTM G2 high-definition mass spectrometer or a Q-Exactive Hybird Quadrupole Qrbitrap mass spectrometer (Thermo Electron Scientific Instrument Crop., WI, United States). Single-crystal X-ray diffraction measurements were conducted on a Bruker Smart Apex II diffractometer (Bruker BioSpin, Zurich, Switzerland) with a graphite monochromator. For preparative HPLC, a Waters 515 HPLC pump, equipped with a Shodex RI-101 Differential Refractometer detector and a JASCO UV-970 intelligent UV/VIS detector, was used. RP-HPLC separations were also conducted using an Shimadzu LC-6AD liquid chromatograph with a SPD-20A UV detector equipped with a YMC Pack ODS-A column $(250 \times 20 \mathrm{~mm}, 120 \AA, 5 \mu \mathrm{m})$. Silica gel $\mathrm{GF}_{254}$ (Qingdao Marine Chemical Factory, P. R. China) was used for TLC. Column chromatography (CC) was performed on Silica gel (200-300 mesh, Qingdao Marine Chemical Factory, P. R. China), Octadecyl silica gel (Merck Chemical Company Ltd., Germany), and Sephadex LH-20 (Amersham Pharmacia Biotech AB, Sweden). All reagents were of analytical grade (Concord Technology Co. Ltd., Tianjin, P. R. China).

\section{Plant Material}

The gum resin of Boswellia sacra Flueck. syn. Boswellia bhawdajiana Birdw. originated in Ethiopia, was furnished by Tianjin Tongrentang Group Co., Ltd. The resin was authenticated by Professor Lin Ma (Tianjin University of Traditional Chinese Medicine). The voucher specimen (accession number: 11037Q) was deposited in the School of Chinese Materia Medica, Tianjin University of Traditional Chinese Medicine, P. R. China.

\section{Extraction and Isolation}

The gum resin of $B$. sacra $(6.8 \mathrm{~kg})$ was powdered and extracted with $95 \% \mathrm{EtOH}$, then the extracts was evaporated to yield a residue $(2.5 \mathrm{~kg})$. The residue was separated by silica gel $\mathrm{CC}$, and eluted sequentially with $\mathrm{PE}$ (petroleum ether), $\mathrm{CH}_{2} \mathrm{Cl}_{2}$ and $\mathrm{MeOH}$. The $\mathrm{CH}_{2} \mathrm{Cl}_{2}$ fraction (513 g) was subjected to silica gel $\mathrm{CC}$ and eluted with a gradient of PE-EtOAc-MeOH to afford ten fractions (A-I).
Fraction B (12.9 g) was further subjected to an ODS CC, and eluted with a gradient of $\mathrm{MeOH}-\mathrm{H}_{2} \mathrm{O}$ to afford subfractions B-1 to B-4. Subfraction B-3 (457 mg) was separated by preparativeHPLC with $\mathrm{MeOH}-\mathrm{H}_{2} \mathrm{O}$ (9:1) to afford compound 5 (2.0 mg).

Fraction C (12.2 g) was subjected to ODS CC, and eluted with a gradient of $\mathrm{MeOH}-\mathrm{H}_{2} \mathrm{O}$ to afford subfraction C-2. Subfraction C-2 (2.1 g) was subjected to Sephadex LH-20 CC and eluted with $\mathrm{CH}_{2} \mathrm{Cl}_{2}-\mathrm{MeOH}$ (1:1) to afford subfractions C-2-1 and C-2-2. Subfraction C-2-2 was separated by preparative-HPLC with $\mathrm{MeOH}-\mathrm{H}_{2} \mathrm{O}$ (9:1) to afford compound 2 (15.4 mg).

Fraction D (23.5 g) was subjected to ODS CC and eluted with a gradient of $\mathrm{MeOH}-\mathrm{H}_{2} \mathrm{O}$ to afford subfractions $\mathrm{D}-1$ to $\mathrm{D}-7$. Subfraction D-3 $(651 \mathrm{mg})$ was separated by preparative-HPLC with $\mathrm{MeCN}-\mathrm{H}_{2} \mathrm{O}$ (7:3) to afford compounds 9 (38.9 mg). Subfraction D-5 (1.2 g) was subjected to Sephadex LH-20 CC and eluted with $\mathrm{CH}_{2} \mathrm{Cl}_{2}-\mathrm{MeOH}$ (1:1), then the subfraction was separated by preparative-HPLC with $\mathrm{MeCN}-\mathrm{H}_{2} \mathrm{O}$ (13:7) to afford compound $6(4.0 \mathrm{mg})$. Subfraction D-6 $(504 \mathrm{mg})$ was subjected to Sephadex LH-20 CC and eluted with $\mathrm{CH}_{2} \mathrm{Cl}_{2}-\mathrm{MeOH}$ (1:1), and then the subfraction was separated by preparative-HPLC with $\mathrm{MeCN}-\mathrm{H}_{2} \mathrm{O}$ (7:3) to afford compound 4 (2.7 mg). Subfraction D$7(336 \mathrm{mg})$ was separated by preparative-HPLC with $\mathrm{MeCN}-\mathrm{H}_{2} \mathrm{O}$ (7:3) to afford compound 7 (2.5 mg).

Fraction F (18.4 g) was subjected to silica gel CC, and eluted with a gradient of PE-EtOAc to afford subfractions F-1 to F-3. Subfraction F-3 (1.1 g) was subjected to silica gel CC, eluted with PE-Acetone (4:1), and then separated by preparative-HPLC with $\mathrm{MeOH}-\mathrm{H}_{2} \mathrm{O}$ (17:3) to afford compound 3 (11.3 mg).

Fraction $\mathrm{H}(8.3 \mathrm{~g})$ was subjected to ODS CC, and eluted with a gradient of $\mathrm{MeOH}-\mathrm{H}_{2} \mathrm{O}$ to afford subfractions $\mathrm{H}-1$ and $\mathrm{H}-2$. Subfraction H-2 (1.3 g) was subjected to Sephadex LH-20 CC, eluted with $\mathrm{MeOH}$, and then separated by preparative-HPLC with $\mathrm{MeCN}-\mathrm{H}_{2} \mathrm{O}$ (9:11) to afford compound 8 (9.4 mg).

Fraction I (10.6 g) was subjected to ODS CC and eluted with a gradient of $\mathrm{MeOH}-\mathrm{H}_{2} \mathrm{O}$ to afford subfractions I-1 to I-3. Subfraction I-2 (2.5 g) was subjected to Sephadex LH-20 CC, eluted with $\mathrm{CH}_{2} \mathrm{Cl}_{2}-\mathrm{MeOH}(1: 1)$, and then separated by preparative-HPLC with $\mathrm{MeOH}-\mathrm{H}_{2} \mathrm{O}(7: 3)$ to afford compound 1 (17.6 mg).

Sacraoxide A (1), colorless needles; mp $161-162^{\circ} \mathrm{C}[\alpha]_{\mathrm{D}}^{25}+15.0$ (c 0.12, MeOH); UV (MeOH) $\lambda_{\max }(\log \varepsilon) 201$ (3.88) nm; ECD (c $\left.1.6 \times 10^{-4} \mathrm{M}, \mathrm{MeOH}\right) \lambda_{\max }(\Delta \varepsilon) 204(+30.89) \mathrm{nm}$; IR (film) $\nu_{\max }$ 3357, 2959, 2921, 1655, 1448, 1383, 1089, 1061, 1033, 1021, 928, $892 \mathrm{~cm}^{-1} ;{ }^{1} \mathrm{H}$ and ${ }^{13} \mathrm{C}$ NMR spectroscopic data, see Tables 1, 2; HRESIMS $m / z$ 323.2574 $[\mathrm{M}+\mathrm{H}]^{+}$(calcd for $\mathrm{C}_{20} \mathrm{H}_{35} \mathrm{O}_{3}$, 323.2581).

Sacraoxide B (3), colorless oil $[\alpha]_{\mathrm{D}}^{25}-4.1(c$ 0.10, MeOH); UV $(\mathrm{MeOH}) \lambda_{\max }(\log \varepsilon) 202(3.31) \mathrm{nm} ; \mathrm{ECD}\left(c 1.6 \times 10^{-4} \mathrm{M}, \mathrm{MeOH}\right)$ $\lambda_{\text {max }}(\Delta \varepsilon) 195(-5.62), 206(+9.27) \mathrm{nm}$; IR (film) $\nu_{\max } 3467,2958$, $2928,1726,1448,1372,1236,1099,1027,925,890 \mathrm{~cm}^{-1} ;{ }^{1} \mathrm{H}$ and ${ }^{13} \mathrm{C}$ NMR spectroscopic data, see Tables 1, 2; HRESIMS $\mathrm{m} / \mathrm{z}$ $387.2513[\mathrm{M}+\mathrm{Na}]^{+}$(calcd for $\mathrm{C}_{22} \mathrm{H}_{36} \mathrm{O}_{4} \mathrm{Na}, 387.2511$ ).

Sacraoxide C (4), colorless oil $[\alpha]_{\mathrm{D}}^{25}+10.4(c 0.10, \mathrm{MeOH})$; UV $(\mathrm{MeOH}) \lambda_{\max }(\log \varepsilon) 201(3.18) \mathrm{nm} ; \mathrm{ECD}\left(c 7.8 \times 10^{-5} \mathrm{M}, \mathrm{MeOH}\right)$ $\lambda_{\max }(\Delta \varepsilon) 205(+1.02), 239(-0.04), 292(+0.49) \mathrm{nm}$; IR (film) $v_{\max }$ $3417,2965,2932,2875,1735,1732,1457,1372,1310,1239,1025$, 1023, 923, $886 \mathrm{~cm}^{-1} ;{ }^{1} \mathrm{H}$ and ${ }^{13} \mathrm{C}$ NMR spectroscopic data, see 
TABLE 1| ${ }^{1} \mathrm{H}$ NMR spectroscopic data of compounds 1, 3-5 ( $\delta$ in ppm, and $J$ in $\mathrm{Hz}$, in $\left.\mathrm{CDCl}_{3}\right)$.

\begin{tabular}{|c|c|c|c|c|}
\hline Position & $1^{a}$ & $3^{b}$ & $4^{b}$ & $5^{b}$ \\
\hline \multirow[t]{2}{*}{2} & $2.38, \mathrm{dd},(15.8,8.5), \beta$ & $2.52, \mathrm{dd},(14.4,10.2), \beta$ & 2.63, d (14.4), $\beta$ & $2.91, \mathrm{~d},(12.6), \beta$ \\
\hline & $1.93^{\mathrm{C}}, \alpha$ & 1.89, dd, (14.4, 4.8), $\alpha$ & 2.57, d (14.4), $\alpha$ & 2.34, d, (12.6), $\alpha$ \\
\hline 3 & $5.27, \mathrm{dd},(8.5,7.4)$ & $5.40, \mathrm{dd},(10.2,4.8)$ & & \\
\hline 4 & & & $2.56, \mathrm{~m}, \alpha$ & $2.91, \mathrm{~m}, \alpha$ \\
\hline \multirow[t]{2}{*}{5} & 2.22, ddd, (14.0, 11.5, 2.3), $\alpha$ & 2.39, td, (13.2, 3.6), $\alpha$ & $1.87, \mathrm{~m}, \alpha$ & $1.65^{\mathrm{c}}, \beta$ \\
\hline & $2.05^{\mathrm{C}}, \beta$ & 2.08, brd, (13.2), $\beta$ & $1.35, \mathrm{~m}, \beta$ & $1.23, \mathrm{~m}, \beta$ \\
\hline \multirow[t]{2}{*}{6} & 1.74, ddd $(11.5,7.8,1.4), \beta$ & 1.70, ddd, (13.2, 9.6, 3.6), $\alpha$ & $2.12, \mathrm{~m}, \alpha$ & $2.19, \mathrm{~m}, \alpha$ \\
\hline & 1.53, ddt, (14.0, 11.2, 3.2), $\alpha$ & 1.36, ddd, $(13.2,13.2,1.2), \beta$ & $2.10, \mathrm{~m}, \beta$ & $2.08^{\mathrm{C}}, \beta$ \\
\hline 7 & $4.63, \mathrm{dd},(7.8,3.2)$ & $4.93, \mathrm{dd},(9.6,1.2)$ & $5.08, \mathrm{t},(7.2)$ & 5.42, dd, $(9.6,6.0)$ \\
\hline \multirow[t]{2}{*}{9} & $5.34, \mathrm{t},(6.6)$ & 5.09, ddd, $(9.6,5.4,1.2)$ & $2.24, \mathrm{~m}, \alpha$ & $1.98, \mathrm{~m}, \alpha$ \\
\hline & & & $1.97, \mathrm{~m}, \beta$ & 1.80, td, $(13.8,1.8), \beta$ \\
\hline \multirow[t]{2}{*}{10} & 2.30, ddd, (15.8, 7.2, 2.6), $\alpha$ & $2.53^{\mathrm{C}}, \alpha$ & $1.99, \mathrm{~m}, \alpha$ & $1.68, \mathrm{~m}, \alpha$ \\
\hline & $2.09, \mathrm{dd},(15.8,11.2), \beta$ & 1.88, dd, $(14.4,12.0), \beta$ & $1.42, \mathrm{~m}, \beta$ & $1.65^{\mathrm{c}}, \beta$ \\
\hline 11 & $3.45, \mathrm{dd},(11.2,2.6), \alpha$ & $5.13, \mathrm{dd},(12.0,1.8), \alpha$ & 3.44, d, (10.2), $\alpha$ & 5.13, d, (9.0), $\alpha$ \\
\hline \multirow[t]{2}{*}{13} & $1.95, \mathrm{~m}, \alpha$ & $1.72, \mathrm{~m}, \alpha$ & $1.97, \mathrm{~m}, \alpha$ & $1.94, \mathrm{~m}, \alpha$ \\
\hline & $1.78, \mathrm{~m}, \beta$ & $1.69, \mathrm{~m}, \beta$ & $1.80, \mathrm{~m}, \beta$ & $1.60, \mathrm{~m}, \beta$ \\
\hline \multirow[t]{2}{*}{14} & $1.83, \mathrm{~m}, \alpha$ & $2.01, \mathrm{td},(12.0,7.8), \alpha$ & $1.48, \mathrm{~m}, \beta$ & $1.95, \mathrm{~m}, \beta$ \\
\hline & $1.78, \mathrm{~m}, \beta$ & $1.81, \mathrm{~m}, \beta$ & $1.37, \mathrm{~m}, \alpha$ & $1.52, \mathrm{~m}, \alpha$ \\
\hline 15 & $1.86, \mathrm{~m}$ & $1.84, \mathrm{~m}$ & 2.40, hept, (7.2) & $1.61, \mathrm{~m}$ \\
\hline 16 & $0.98, \mathrm{~d},(6.9)$ & $0.88, \mathrm{~d},(7.2)$ & $0.87, \mathrm{~d},(6.6)$ & $0.91, \mathrm{~d},(6.6)$ \\
\hline 17 & $0.86, \mathrm{~d},(7.2)$ & $1.00, \mathrm{~d},(7.2)$ & $0.90, d,(6.6)$ & $0.87, \mathrm{~d},(6.6)$ \\
\hline 18 & $1.60, \mathrm{~s}$ & $1.59, \mathrm{~s}$ & $1.04, \mathrm{~d},(7.2)$ & $1.03, \mathrm{~d},(6.0)$ \\
\hline 19 & $1.70, \mathrm{~s}$ & $1.65, \mathrm{~s}$ & $1.67, \mathrm{~s}$ & $1.66, \mathrm{~s}$ \\
\hline 20 & $1.22, \mathrm{~s}$ & $1.22, \mathrm{~s}$ & $1.09, \mathrm{~s}$ & $1.11, \mathrm{~s}$ \\
\hline Ac- $2^{\prime}$ & & $2.03, \mathrm{~s}$ & & $2.09, \mathrm{~s}$ \\
\hline
\end{tabular}

${ }^{a}$ Recorded at $500 \mathrm{MHz}$.

${ }^{b}$ Recorded at $600 \mathrm{MHz}$.

${ }^{c}$ Overlapping resonances.

TABLE $2 \mid{ }^{13} \mathrm{C}$ NMR spectroscopic data of compounds 1, 3-8 $\left(\delta\right.$ in ppm, in $\left.\mathrm{CDCl}_{3}\right)$.

\begin{tabular}{|c|c|c|c|c|c|c|c|}
\hline Position & $1^{a}$ & $3^{b}$ & $4^{b}$ & $5^{b}$ & $6^{b}$ & $7^{b}$ & $8^{a}$ \\
\hline 1 & $88.5, \mathrm{C}$ & $89.5, \mathrm{C}$ & $88.9, \mathrm{C}$ & 89.6, C & $89.3, \mathrm{C}$ & $89.2, \mathrm{C}$ & $88.2, \mathrm{C}$ \\
\hline 2 & 28.5, $\mathrm{CH}_{2}$ & 28.1, $\mathrm{CH}_{2}$ & 47.3, $\mathrm{CH}_{2}$ & 49.6, $\mathrm{CH}_{2}$ & 34.0, $\mathrm{CH}_{2}$ & 33.1, $\mathrm{CH}_{2}$ & 128.8, CH \\
\hline 3 & $121.9, \mathrm{CH}$ & 121.8, CH & 213.4, C & $215.7, \mathrm{C}$ & $126.7, \mathrm{C}$ & 123.2, $\mathrm{CH}$ & 137.6, $\mathrm{CH}$ \\
\hline 4 & 137.0, C & 137.1, C & $46.5, \mathrm{CH}$ & $42.5, \mathrm{CH}$ & $131.5, \mathrm{C}$ & $134.4, \mathrm{C}$ & $74.4, \mathrm{C}$ \\
\hline 5 & 35.6, $\mathrm{CH}_{2}$ & $36.2, \mathrm{CH}_{2}$ & $30.8, \mathrm{CH}_{2}$ & $33.4, \mathrm{CH}_{2}$ & $57.1, \mathrm{CH}_{2}$ & $38.5, \mathrm{CH}_{2}$ & 43.8, $\mathrm{CH}_{2}$ \\
\hline 6 & 32.6, $\mathrm{CH}_{2}$ & $30.5, \mathrm{CH}_{2}$ & 25.2, $\mathrm{CH}_{2}$ & 24.6, $\mathrm{CH}_{2}$ & 201.2, C & 24.6, $\mathrm{CH}_{2}$ & 24.3, $\mathrm{CH}_{2}$ \\
\hline 7 & $68.3, \mathrm{CH}$ & $66.1, \mathrm{CH}$ & 124.6, $\mathrm{CH}$ & $125.8, \mathrm{CH}$ & 120.3, CH & $143.7, \mathrm{CH}$ & $128.5, \mathrm{CH}$ \\
\hline 8 & $140.7, \mathrm{C}$ & 143.6, C & 135.6, C & $135.8, \mathrm{C}$ & $154.8, \mathrm{C}$ & $135.7, \mathrm{C}$ & 133.6, C \\
\hline 9 & 121.9, CH & 118.8, CH & $32.5, \mathrm{CH}_{2}$ & $34.1, \mathrm{CH}_{2}$ & 33.8, $\mathrm{CH}_{2}$ & 202.2, C & $35.2, \mathrm{CH}_{2}$ \\
\hline 10 & $28.1, \mathrm{CH}_{2}$ & 25.5, $\mathrm{CH}_{2}$ & $31.8, \mathrm{CH}_{2}$ & 29.1, $\mathrm{CH}_{2}$ & 25.8, $\mathrm{CH}_{2}$ & 40.6, $\mathrm{CH}_{2}$ & 29.6, $\mathrm{CH}_{2}$ \\
\hline 11 & $76.9, \mathrm{CH}$ & $77.2, \mathrm{CH}$ & 77.3, $\mathrm{CH}$ & $78.2, \mathrm{CH}$ & $77.2, \mathrm{CH}$ & $78.2, \mathrm{CH}$ & $76.2, \mathrm{CH}$ \\
\hline 12 & $84.1, \mathrm{C}$ & 82.6, C & $85.5, \mathrm{C}$ & $84.7, \mathrm{C}$ & $83.5, \mathrm{C}$ & $83.2, \mathrm{C}$ & $84.6, \mathrm{C}$ \\
\hline 13 & $35.2, \mathrm{CH}_{2}$ & 35.3, $\mathrm{CH}_{2}$ & $36.5, \mathrm{CH}_{2}$ & 35.3, $\mathrm{CH}_{2}$ & 35.6, $\mathrm{CH}_{2}$ & $35.8, \mathrm{CH}_{2}$ & 36.6, $\mathrm{CH}_{2}$ \\
\hline 14 & $31.2, \mathrm{CH}_{2}$ & 29.6, $\mathrm{CH}_{2}$ & 31.0, $\mathrm{CH}_{2}$ & $31.4, \mathrm{CH}_{2}$ & $30.5, \mathrm{CH}_{2}$ & $30.3, \mathrm{CH}_{2}$ & $34.9, \mathrm{CH}_{2}$ \\
\hline 15 & $35.2, \mathrm{CH}$ & $35.3, \mathrm{CH}$ & 33.1, CH & $35.2, \mathrm{CH}$ & $33.4, \mathrm{CH}$ & $33.1, \mathrm{CH}$ & $35.2, \mathrm{CH}$ \\
\hline 16 & 16.1, $\mathrm{CH}_{3}$ & 19.3, $\mathrm{CH}_{3}$ & 18.7, $\mathrm{CH}_{3}$ & $18.2, \mathrm{CH}_{3}$ & $18.8, \mathrm{CH}_{3}$ & $18.5, \mathrm{CH}_{3}$ & $18.5, \mathrm{CH}_{3}$ \\
\hline 17 & 19.1, $\mathrm{CH}_{3}$ & 16.1, $\mathrm{CH}_{3}$ & 17.3, $\mathrm{CH}_{3}$ & $17.2, \mathrm{CH}_{3}$ & $17.5, \mathrm{CH}_{3}$ & $17.4, \mathrm{CH}_{3}$ & $17.5, \mathrm{CH}_{3}$ \\
\hline 18 & $15.8, \mathrm{CH}_{3}$ & $15.4, \mathrm{CH}_{3}$ & 16.8, $\mathrm{CH}_{3}$ & $14.2, \mathrm{CH}_{3}$ & 16.3, $\mathrm{CH}_{3}$ & $14.8, \mathrm{CH}_{3}$ & 29.2, $\mathrm{CH}_{3}$ \\
\hline 19 & 17.0, $\mathrm{CH}_{3}$ & $16.9, \mathrm{CH}_{3}$ & 19.3, $\mathrm{CH}_{3}$ & 17.0, $\mathrm{CH}_{3}$ & 20.6, $\mathrm{CH}_{3}$ & $11.5, \mathrm{CH}_{3}$ & 16.6, $\mathrm{CH}_{3}$ \\
\hline 20 & 23.6, $\mathrm{CH}_{3}$ & 22.3, $\mathrm{CH}_{3}$ & 19.6, $\mathrm{CH}_{3}$ & $21.2, \mathrm{CH}_{3}$ & 21.3, $\mathrm{CH}_{3}$ & $21.6, \mathrm{CH}_{3}$ & 20.0, $\mathrm{CH}_{3}$ \\
\hline$A c-1^{\prime}$ & & $171.8, \mathrm{C}$ & & 171.3, C & $171.0, \mathrm{C}$ & $170.4, \mathrm{C}$ & \\
\hline$A c-2^{\prime}$ & & 21.3, $\mathrm{CH}_{3}$ & & 21.3, $\mathrm{CH}_{3}$ & $21.1, \mathrm{CH}_{3}$ & $21.0, \mathrm{CH}_{3}$ & \\
\hline
\end{tabular}

${ }^{a}$ Recorded at $125 \mathrm{MHz}$.

${ }^{b}$ Recorded at $150 \mathrm{MHz}$

Tables 1, 2; HRESIMS $\mathrm{m} / \mathrm{z} 323.2581[\mathrm{M}+\mathrm{H}]^{+}$(calcd for $\mathrm{C}_{20} \mathrm{H}_{35} \mathrm{O}_{3}, 323.2586$ ); $/ z / z 345.2401[\mathrm{M}+\mathrm{Na}]^{+}$(calcd for $\mathrm{C}_{20} \mathrm{H}_{34} \mathrm{O}_{3} \mathrm{Na}, 345.2406$ ).
Sacraoxide D (5), pale yellow oil $[\alpha]_{\mathrm{D}}^{25}+47.7($ c 0.10, MeOH); $\mathrm{UV}(\mathrm{MeOH}) \lambda_{\max }(\log \varepsilon) 201(3.09) \mathrm{nm} ; \mathrm{ECD}\left(c 6.8 \times 10^{-5} \mathrm{M}\right.$, $\mathrm{MeOH}) \lambda_{\max }(\Delta \varepsilon) 207(+1.48), 237(-0.00), 295(+2.00) \mathrm{nm}$; IR 
TABLE 3 $\mid{ }^{1} \mathrm{H}$ NMR spectroscopic data of compounds 6-8 ( $\delta$ in ppm, and $J$ in $\mathrm{Hz}$, in $\left.\mathrm{CDCl}_{3}\right)$.

\begin{tabular}{|c|c|c|c|}
\hline Position & $6^{a}$ & $7^{a}$ & $8^{b}$ \\
\hline 2 & $\begin{array}{l}\text { 2.30, dd, }(12.6,4.2), \beta \\
1.85, \mathrm{dd},(12.6,7.2), \alpha\end{array}$ & $\begin{array}{l}2.29, \mathrm{dd},(12.6,4.2), \beta \\
2.10, \mathrm{dd},(12.6,5.4), a\end{array}$ & $5.54, d,(15.4)$ \\
\hline 3 & $5.49, \mathrm{dd},(7.2,4.2)$ & $5.35, \mathrm{dd},(5.4,4.2)$ & $5.81, d,(15.4)$ \\
\hline 5 & $\begin{array}{l}\text { 3.10, d, (14.4), } \alpha \\
2.92, d,(14.4), \beta\end{array}$ & $\begin{array}{l}2.31^{c}, a \\
2.30^{c}, \beta\end{array}$ & $\begin{array}{l}1.82^{\mathrm{C}}, \alpha \\
1.57, \mathrm{td},(11.4,1.4), \beta\end{array}$ \\
\hline 6 & & $\begin{array}{l}2.51, d d, 1(2.6,9.6), \alpha \\
2.27, d d,(12.6,1.8), \beta\end{array}$ & $\begin{array}{l}2.23, \mathrm{ddd},(15.2,4.2,1.4), \alpha \\
2.15, \mathrm{dd},(15.2,10.2), \beta\end{array}$ \\
\hline 7 & $6.53, \mathrm{~s}$ & $6.68, \mathrm{dd},(9.6,1.8)$ & $5.16, \mathrm{dd},(10.2,4.2)$ \\
\hline 9 & $\begin{array}{l}2.27^{\mathrm{c}}, \alpha \\
1.82, \mathrm{dd},(12.6,7.2), \beta\end{array}$ & & $2.07, \mathrm{~m}$ \\
\hline 10 & $\begin{array}{l}2.10, \mathrm{~d},(14.4), \alpha \\
1.60^{\mathrm{c}}, \beta\end{array}$ & $\begin{array}{l}3.60, \mathrm{dd},(12.6,0.6), \alpha \\
2.25, \mathrm{dd},(12.6,10.8), \beta\end{array}$ & $\begin{array}{l}\text { 1.93, ddd, }(15.8,7.4,1.8), \alpha \\
1.31, d d,(15.8,9.8), \beta\end{array}$ \\
\hline 11 & 4.84, d, (10.8), $\alpha$ & $4.87, \mathrm{dd},(10.8,0.6), a$ & $3.47, d,(9.8), \alpha$ \\
\hline 13 & $\begin{array}{l}1.88, \mathrm{~m}, \alpha \\
1.64, \mathrm{~m}, \beta\end{array}$ & $\begin{array}{l}1.82, \text { ddd, }(12.6,10.8,4.2), \alpha \\
1.69^{c}, \beta\end{array}$ & $\begin{array}{l}1.81, \mathrm{~m}, \mathrm{a} \\
1.72, \mathrm{~m}, \beta\end{array}$ \\
\hline 14 & $\begin{array}{l}1.89, \mathrm{~m}, \beta \\
1.49, \mathrm{~m}, \alpha\end{array}$ & $\begin{array}{l}\text { 1.90, ddd, }(12.6,10.8,4.2), \beta \\
1.53, \text { ddd, }(12.6,7.8,4.2), \alpha\end{array}$ & $\begin{array}{l}\text { 1.88, dd, }(11.2,7.4), \beta \\
1.83, \mathrm{~m}, \alpha\end{array}$ \\
\hline 15 & $2.10, \mathrm{~m}$ & 2.21 , hept, $(7.2)$ & 1.64, hept, (6.9) \\
\hline 16 & $0.93, \mathrm{~d},(6.6)$ & $0.91, d,(6.6)$ & $0.82, d,(7.1)$ \\
\hline 17 & $0.96, \mathrm{~d},(6.6)$ & $0.94, \mathrm{~d},(6.6)$ & $0.79, \mathrm{~d},(6.5)$ \\
\hline 18 & $1.60, \mathrm{~s}$ & $1.57, \mathrm{~s}$ & $1.27, \mathrm{~s}$ \\
\hline 19 & $2.05, \mathrm{~s}$ & $1.69, \mathrm{~s}$ & $1.66, \mathrm{~s}$ \\
\hline 20 & $1.12, \mathrm{~s}$ & $1.13, \mathrm{~s}$ & $1.04, \mathrm{~s}$ \\
\hline Ac- $2^{\prime}$ & $2.05, \mathrm{~s}$ & $1.99, \mathrm{~s}$ & \\
\hline
\end{tabular}

${ }^{a}$ Recorded at $600 \mathrm{MHz}$.

${ }^{b}$ Recorded at $500 \mathrm{MHz}$

${ }^{c}$ Overlapping resonances.

(film) $v_{\max } 2965,2931,2875,1732,1728,1457,1372,1238,1110$, 1023, 918, $886 \mathrm{~cm}^{-1} ;{ }^{1} \mathrm{H}$ and ${ }^{13} \mathrm{C}$ NMR spectroscopic data, see Tables 1, 2; HRESIMS $m / z$ 387.2515 $[\mathrm{M}+\mathrm{Na}]^{+}$(calcd for $\left.\mathrm{C}_{22} \mathrm{H}_{36} \mathrm{O}_{4} \mathrm{Na}, 387.2511\right)$.

Sacraoxide E (6), colorless oil $[\alpha]_{\mathrm{D}}^{25}-64.0($ c $0.10, \mathrm{MeOH})$; UV $(\mathrm{MeOH}) \lambda_{\max }(\log \varepsilon) 201$ (3.38), $234(3.08) \mathrm{nm} ; \mathrm{ECD}\left(c 6.9 \times 10^{-}\right.$ $\left.{ }^{5} \mathrm{M}, \mathrm{MeOH}\right) \lambda_{\max }(\Delta \varepsilon) 203(+0.60), 241(-2.01), 280(+0.02), 348$ $(-0.72) \mathrm{nm}$; IR (film) $\nu_{\max } 2960,2926,1733,1684,1373,1236$, 1240, 1097, 1039, 976, $891 \mathrm{~cm}^{-1} ;{ }^{1} \mathrm{H}$ and ${ }^{13} \mathrm{C}$ NMR spectroscopic data, see Tables 2, 3; HRESIMS $m / z$ 385.2354 [M $+\mathrm{Na}]^{+}$(calcd for $\mathrm{C}_{22} \mathrm{H}_{34} \mathrm{O}_{4} \mathrm{Na}$, 385.2355).

Sacraoxide F (7), pale yellow oil $[\alpha]_{\mathrm{D}}^{25}+10.2(c 0.10, \mathrm{MeOH})$; $\mathrm{UV}(\mathrm{MeOH}) \lambda_{\max }(\log \varepsilon) 201$ (3.50), 226 (3.10) nm; ECD (c 7.8 $\left.\times 10^{-5} \mathrm{M}, \mathrm{MeOH}\right) \lambda_{\max }(\Delta \varepsilon) 233(-2.85), 287(+0.18) \mathrm{nm}$; IR (film) $v_{\max } 2960,2929,1733,1684,1374,1236,1028,981$, $854 \mathrm{~cm}^{-1} ;{ }^{1} \mathrm{H}$ and ${ }^{13} \mathrm{C}$ NMR spectroscopic data, see Tables 2, 3; HRESIMS $m / z 363.2504[\mathrm{M}+\mathrm{H}]^{+}$(calcd for $\mathrm{C}_{22} \mathrm{H}_{35} \mathrm{O}_{4}$, 363.2535).

Sacraoxide $\mathrm{G}(\mathbf{8})$, white amorphous powder $[\alpha]_{\mathrm{D}}^{25}+115.0(c$ 0.11, MeOH); UV (MeOH) $\lambda_{\max }(\log \varepsilon) 201$ (3.29) nm; ECD (c 3.1 $\left.\times 10^{-4} \mathrm{M}, \mathrm{MeOH}\right) \lambda_{\max }(\Delta \varepsilon) 203(+22.82) \mathrm{nm} ; \mathrm{IR}\left(\right.$ film) $\nu_{\max } 3358$, $2919,2849,1658,1632,1469,1382,1075,977,944 \mathrm{~cm}^{-1} ;{ }^{1} \mathrm{H}$ and ${ }^{13} \mathrm{C}$ NMR spectroscopic data, see Tables 2, 3; HRESIMS $\mathrm{m} / \mathrm{z}$ $305.2583\left[\mathrm{M}-\mathrm{H}_{2} \mathrm{O}+\mathrm{H}\right]^{+}$(calcd for $\mathrm{C}_{20} \mathrm{H}_{33} \mathrm{O}_{2}, 305.2475$ ).

Boscartins AD (2), colorless oil; ${ }^{1} \mathrm{H}$ NMR $\left(600 \mathrm{MHz}, \mathrm{CDCl}_{3}\right) \delta$ $2.41(1 \mathrm{H}, \mathrm{dd}, J=15.6,6.6 \mathrm{~Hz}, \mathrm{H}-2 \beta), 1.91(1 \mathrm{H}, \mathrm{dd}, J=15.6,4.8 \mathrm{~Hz}$, $\mathrm{H}-2 \alpha), 5.11(1 \mathrm{H}, \mathrm{dd}, J=6.6,4.8 \mathrm{~Hz}, \mathrm{H}-3), 2.15(1 \mathrm{H}, \mathrm{dd}, J=14.4$, $9.0 \mathrm{~Hz}, \mathrm{H}-5 \alpha), 1.94(1 \mathrm{H}$, brd, $J=14.4 \mathrm{~Hz}, \mathrm{H}-5 \beta), 1.83(1 \mathrm{H}$, overlap, H-6 $\alpha), 1.62(1 \mathrm{H}$, overlap, $\mathrm{H}-6 \beta), 4.72(1 \mathrm{H}, \mathrm{t}, J=6.6 \mathrm{~Hz}$,
H-7), 5.57 (1H, dd, $J=12.0,4.2 \mathrm{~Hz}, \mathrm{H}-9), 2.93(1 \mathrm{H}, \mathrm{dd}, J=13.2$, $12.0 \mathrm{~Hz}, \mathrm{H}-10 \alpha), 1.69$ (1H, ddd, $J=13.2,10.8,4.2 \mathrm{~Hz}, \mathrm{H}-10 \beta)$, $3.20(1 \mathrm{H}, \mathrm{d}, J=10.8 \mathrm{~Hz}, \mathrm{H}-11), 2.07(1 \mathrm{H}, \mathrm{m}, \mathrm{H}-13 \alpha), 1.74(1 \mathrm{H}, \mathrm{m}$, $\mathrm{H}-13 \beta), 1.80$ (2H, m, H-14), 1.83 (1H, m, H-15), 0.97 (3H, d, $J=$ $\left.6.6 \mathrm{~Hz}, \mathrm{H}_{3}-16\right), 0.88\left(3 \mathrm{H}, \mathrm{d}, J=7.2 \mathrm{~Hz}, \mathrm{H}_{3}-17\right), 1.62\left(3 \mathrm{H}, \mathrm{s}, \mathrm{H}_{3^{-}}\right.$ 18), $1.74\left(3 \mathrm{H}, \mathrm{s}, \mathrm{H}_{3}-19\right), 1.16\left(3 \mathrm{H}, \mathrm{s}, \mathrm{H}_{3}-20\right) ;{ }^{13} \mathrm{C} \mathrm{NMR}(150 \mathrm{MHz}$, $\left.\mathrm{CDCl}_{3}\right) \delta 88.2(\mathrm{C}-1), 30.1(\mathrm{C}-2), 121.2(\mathrm{C}-3), 134.2(\mathrm{C}-4), 34.7(\mathrm{C}-$ 5), 31.3 (C-6), 66.9 (C-7), 138.0 (C-8), 125.6 (C-9), 29.0 (C-10), 78.4 (C-11), 84.3 (C-12), 35.4 (C-13), 30.9 (C-14), 35.8 (C-15), 17.1 (C-16), 18.8 (C-17), 16.4 (C-18), 17.1 (C-19), 20.8 (C-20); HRESIMS $m / z$ 305.2471 $\left[\mathrm{M}-\mathrm{H}_{2} \mathrm{O}+\mathrm{H}\right]^{+}$(calcd for $\mathrm{C}_{20} \mathrm{H}_{33} \mathrm{O}_{2}$, 305.2475).

Boscartins L (10), pale yellow oil; ${ }^{1} \mathrm{H}$ NMR $\left(600 \mathrm{MHz}, \mathrm{CDCl}_{3}\right)$ $\delta 2.42(1 \mathrm{H}, \mathrm{dd}, J=16.2,7.2 \mathrm{~Hz}, \mathrm{H}-2 \beta), 1.89(1 \mathrm{H}$, overlap, H-2 $\alpha)$, $5.22(1 \mathrm{H}, \mathrm{dd}, J=7.2,5.4 \mathrm{~Hz}, \mathrm{H}-3), 2.15(1 \mathrm{H}, \mathrm{m}, \mathrm{H}-5 \beta), 1.98(1 \mathrm{H}$, $\mathrm{m}, \mathrm{H}-5 \alpha), 1.82(1 \mathrm{H}, \mathrm{m}, \mathrm{H}-6 \alpha), 1.68(1 \mathrm{H}$, overlap, $\mathrm{H}-6 \beta), 4.68(1 \mathrm{H}$, $\mathrm{t}, J=6.6 \mathrm{~Hz}, \mathrm{H}-7), 5.18(1 \mathrm{H}, \mathrm{dd}, J=11.4,4.2 \mathrm{~Hz}, \mathrm{H}-9), 2.90(1 \mathrm{H}$, $\mathrm{dd}, J=13.2,11.4 \mathrm{~Hz}, \mathrm{H}-10 \alpha), 1.88(1 \mathrm{H}$, ddd, $J=13.2,11.4,4.2 \mathrm{~Hz}$, $\mathrm{H}-10 \beta), 4.72(1 \mathrm{H}, \mathrm{d}, J=11.4 \mathrm{~Hz}, \mathrm{H}-11), 1.78(1 \mathrm{H}, \mathrm{m}, \mathrm{H}-13 \alpha), 1.64$

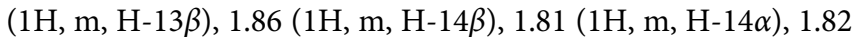
$(1 \mathrm{H}, \mathrm{m}, \mathrm{H}-15), 0.88\left(3 \mathrm{H}, \mathrm{d}, J=7.2 \mathrm{~Hz}, \mathrm{H}_{3}-16\right), 0.96(3 \mathrm{H}, \mathrm{d}, J=7.2$ $\left.\mathrm{Hz}, \mathrm{H}_{3}-17\right), 1.65$ (3H, s, $\left.\mathrm{H}_{3}-18\right), 1.68$ (3H, s, $\left.\mathrm{H}_{3}-19\right), 1.18$ (3H, s, $\left.\mathrm{H}_{3}-20\right), 2.09\left(3 \mathrm{H}, \mathrm{s}, \mathrm{H}_{3}-2^{\prime}\right) ;{ }^{13} \mathrm{C} \mathrm{NMR}\left(150 \mathrm{MHz}, \mathrm{CDCl}_{3}\right) \delta 88.7$ (C-1), 30.3 (C-2), 121.1 (C-3), 134.6 (C-4), 34.9 (C-5), 31.3 (C-6), 67.1 (C-7), 138.1 (C-8), 124.1 (C-9), 26.6 (C-10), 78.0 (C-11), 83.3 (C-12), 35.0 (C-13), 30.9 (C-14), 36.0 (C-15), 18.8 (C-16), 17.1 (C-17), 16.1 (C-18), 17.0 (C-19), 21.8 (C-20), 171.1 (C-1'), 21.3 $\left(\mathrm{C}-2^{\prime}\right)$; HRESIMS $m / z 387.2506[\mathrm{M}+\mathrm{Na}]^{+}$(calcd for $\mathrm{C}_{22} \mathrm{H}_{36} \mathrm{O}_{4} \mathrm{Na}$, 387.2511). 


\section{Crystal Structure Determination of 1}

Crystal Data for $\mathrm{C}_{20} \mathrm{H}_{36} \mathrm{O}_{4}(M=340.49 \mathrm{~g} / \mathrm{mol})$ : monoclinic, space group I2 (no. 5), $a=23.3651$ (6) $\AA, b=10.1181$ (2) $\AA, c=67.6869$ (15) $\AA, \beta=91.113$ (2), $V=15,998.9$ (6) $\AA 3, Z=32, T=100.01$ (11) $\mathrm{K}, \mu(\mathrm{Cu} \mathrm{Ka})=0.608 \mathrm{~mm}^{-1}$, Dcalc $=1.131 \mathrm{~g} / \mathrm{cm}^{3}, 58,130$ reflections measured $(4.026 \leq 2 \theta \leq 148.16)$, 25,373 unique $\left(R_{\text {int }}=0.1124, R_{\text {sigma }}=0.1243\right)$ which were used in all calculations. The final $\mathrm{R}_{1}$ was $0.0818[\mathrm{I}>2 \sigma(\mathrm{I})]$ and $w R_{2}$ was 0.2253 (all data).

\section{Preparation of (R)- and (S)-MTPA Esters of 2}

As described in previous literature (Li et al., 2007), compound $\mathbf{2}$ $(2.0 \mathrm{mg})$ was dissolved in anhydrous pyridine $(0.5 \mathrm{ml})$ and transferred into a dried bottle, and then $10 \mu \mathrm{L}$ of $S$ (-)- $\alpha$-methoxy- $\alpha$-(trifluoromethyl) phenylacetyl chloride (S-MTPA-Cl; Sigma-Aldrich Co., St. Louis, MO, United States) was added. After stirring at room temperature for $24 \mathrm{~h}$, the reaction mixture was evaporated to give a residue, which was purified by semipreparative $\mathrm{HPLC}\left(\mathrm{MeOH}-\mathrm{H}_{2} \mathrm{O}\right.$ 9:1, $\mathrm{v} / \mathrm{v}, 3 \mathrm{ml} / \mathrm{min})$ to give the $R$-MTPA ester derivative of $\mathbf{2}\left(\mathbf{2 a}, t_{\mathrm{R}}=\right.$ $10.2 \mathrm{~min}, 2.1 \mathrm{mg})$. The $S$-MTPA ester derivative of $\mathbf{2}(\mathbf{2} \mathbf{b}, 1.8 \mathrm{mg})$ was obtained by using the same procedure as described above but using $\mathrm{R}$-MTPA-Cl.

$(R)$-MTPA ester of 2 (2a), colorless oil; ${ }^{1} \mathrm{H}$ NMR $(600 \mathrm{MHz}$, $\left.\mathrm{CDCl}_{3}\right) \delta 2.45(1 \mathrm{H}, \mathrm{dd}, J=15.6,7.2 \mathrm{~Hz}, \mathrm{H}-2 \mathrm{a}), 1.87(1 \mathrm{H}$, overlap, H-2b), 5.16 (1H, dd, $J=7.2,5.4 \mathrm{~Hz}, \mathrm{H}-3), 1.97$ (1H, m, H-5a), 1.94 (1H, m, H-5b), 1.97 (1H, m, H-6a), 1.70 (1H, m, H-6b), $5.96(1 \mathrm{H}$, dd, $J=7.2,6.6 \mathrm{~Hz}, \mathrm{H}-7), 5.72(1 \mathrm{H}, \mathrm{dd}, J=12.0,3.6 \mathrm{~Hz}, \mathrm{H}-9), 3.14$ (1H, dd, $J=13.2,12.0 \mathrm{~Hz}, \mathrm{H}-10 \mathrm{a}), 1.77$ (1H, overlap, H-10b), 3.19 $(1 \mathrm{H}, \mathrm{d}, J=10.8 \mathrm{~Hz}, \mathrm{H}-11), 2.05(1 \mathrm{H}, \mathrm{m}, \mathrm{H}-13 \mathrm{a}), 1.75(1 \mathrm{H}, \mathrm{m}, \mathrm{H}-$ 13b), 1.83 (2H, m, H-14), 1.81 (1H, m, H-15), 1.00 (3H, d, J = 7.2 $\left.\mathrm{Hz}, \mathrm{H}_{3}-16\right), 0.89\left(3 \mathrm{H}, \mathrm{d}, J=6.6 \mathrm{~Hz}, \mathrm{H}_{3}-17\right), 1.62\left(3 \mathrm{H}, \mathrm{s}, \mathrm{H}_{3}-18\right)$, 1.69 (3H, s, $\left.\mathrm{H}_{3}-19\right), 1.17$ (3H, s, $\left.\mathrm{H}_{3}-20\right)$; HRESIMS $m / z$ 561.2809 $[\mathrm{M}+\mathrm{Na}]^{+}$(calcd for $\mathrm{C}_{30} \mathrm{H}_{41} \mathrm{O}_{5} \mathrm{~F}_{3} \mathrm{Na}, 561.2804$ ).

(S)-MTPA ester of 2 (2b), colorless oil; ${ }^{1} \mathrm{H}$ NMR $(600 \mathrm{MHz}$, $\left.\mathrm{CDCl}_{3}\right) \delta: 2.45(1 \mathrm{H}, \mathrm{dd}, J=16.2,7.2 \mathrm{~Hz}, \mathrm{H}-2 \mathrm{a}), 1.88(1 \mathrm{H}, \mathrm{dd}, J=$ 16.2, $5.4 \mathrm{~Hz}, \mathrm{H}-2 \mathrm{~b}), 5.16(1 \mathrm{H}, \mathrm{dd}, J=7.2,5.4 \mathrm{~Hz}, \mathrm{H}-3), 2.07(1 \mathrm{H}$, m, H-5a), 1.99 (1H, m, H-5b), 2.05 (1H, m, H-6a), 1.78 (1H, m, H-6b), $5.91(1 \mathrm{H}, \mathrm{dd}, J=6.6,6.0 \mathrm{~Hz}, \mathrm{H}-7), 5.69(1 \mathrm{H}, \mathrm{dd}, J=12.0$, $3.6 \mathrm{~Hz}, \mathrm{H}-9), 3.14$ (1H, dd, $J=13.2,12.0 \mathrm{~Hz}, \mathrm{H}-10 \mathrm{a}), 1.78(1 \mathrm{H}$, ddd, $J=13.2,10.8,4.2 \mathrm{~Hz}, \mathrm{H}-10 \mathrm{~b}), 3.19(1 \mathrm{H}, \mathrm{d}, J=10.8 \mathrm{~Hz}, \mathrm{H}-11)$, $2.06(1 \mathrm{H}, \mathrm{m}, \mathrm{H}-13 \mathrm{a}), 1.74$ (1H, m, H-13b), 1.83 (2H, m, H-14), $1.81(1 \mathrm{H}, \mathrm{m}, \mathrm{H}-15), 0.99\left(3 \mathrm{H}, \mathrm{d}, J=6.6 \mathrm{~Hz}, \mathrm{H}_{3}-16\right), 0.89(3 \mathrm{H}, \mathrm{d}$, $\left.J=7.2 \mathrm{~Hz}, \mathrm{H}_{3}-17\right), 1.50$ (3H, s, $\left.\mathrm{H}_{3}-18\right), 1.63$ (3H, s, $\left.\mathrm{H}_{3}-19\right), 1.17$ $\left(3 \mathrm{H}, \mathrm{s}, \mathrm{H}_{3}-20\right)$; HRESIMS $m / z 561.2804[\mathrm{M}+\mathrm{Na}]^{+}$(calcd for $\left.\mathrm{C}_{30} \mathrm{H}_{41} \mathrm{O}_{5} \mathrm{~F}_{3} \mathrm{Na}, 561.2804\right)$.

\section{ECD Calculations}

The details of the quantum chemical ECD calculations for compounds 4, 6-8 are provided in Supplementary data.

\section{NO Inhibitory Assay}

The RAW 264.7 (ATCCTIB-71) mouse monocyte-macrophages were cultured in RPMI 1640 medium supplemented with penicillin G (100 units $/ \mathrm{mL})$, streptomycin $(100 \mathrm{mg} / \mathrm{ml})$ and $10 \%$ FBS. The cells were seeded in 96-well plastic plates with $1 \times 10^{5}$ cells/well and allowed to adhere for $24 \mathrm{~h}$ at $37^{\circ} \mathrm{C}$ in a humidified atmosphere containing $5 \% \mathrm{CO}_{2}$. Then the medium was replaced with fresh medium, containing LPS $(1 \mu \mathrm{g} / \mathrm{ml})$ together with test compounds at various concentrations and then incubated for $24 \mathrm{~h}$. NO production was determined by measuring the accumulation of nitrite in the culture supernatant using Griess reagent. Briefly, $100 \mu \mathrm{L}$ of the supernatant from incubates were mixed with equal volume of Griess reagent ( $1 \%$ sulfanilamide and $0.1 \%$ naphthylene-diamide dihydrochloride in $2.5 \% \mathrm{H}_{3} \mathrm{PO}_{4}$ ) and were allowed to stand for $10 \mathrm{~min}$ at $37^{\circ} \mathrm{C}$ in a humidified atmosphere containing $5 \% \mathrm{CO}_{2}$. Absorbance at $540 \mathrm{~nm}$ was measured using microplate reader. The nitrite concentrations were calculated according to the literature (Jin et al., 2016).

\section{Cell Viability}

Cell viability was determined using the mitochondrial respiration-dependent MTT reduction method. After transferring the required supernatant to another plate for the Griess assay, the remaining supernatant was aspirated from the 96-well plates, $100 \mu \mathrm{L}$ of fresh medium and $10 \mu \mathrm{L}$ of MTT ( $5 \mathrm{mg} /$ $\mathrm{ml} \mathrm{PBS}$ ) were added to each well. The cells were incubated at $37^{\circ} \mathrm{C}$ in a humidified atmosphere containing $5 \% \mathrm{CO}_{2}$. After incubating for $4 \mathrm{~h}$, the medium was removed and the violet crystals of formazan in viable cells were dissolved in DMSO. Absorbance at $570 \mathrm{~nm}$ was measured using a microplate reader.

\section{RESULTS}

A $95 \% \mathrm{EtOH}$ extract of the gum resin of B. sacra was separated by multiple column chromatography (CC) including silica gel, Sephadex LH-20 and ODS CC, as well as preparative HPLC to afford nine cembranoids (1-9, Figure 1). The known compounds were identified as boscartin $\mathrm{AD}$ (2) and boscartin L (9) by detailed spectroscopic and physicochemical analyses and comparison of literature data (Wang J. J. et al., 2019; Wang Y. G. et al., 2019). The new compounds were named as sacraoxides A-G $(\mathbf{1}, \mathbf{3}-\mathbf{8})$, and their structures were elucidated as follows.

Sacraoxide A (1) was isolated as colorless needles $(\mathrm{MeOH}-$ $\left.\mathrm{H}_{2} \mathrm{O}\right)[\alpha]_{\mathrm{D}}^{25}+15.0(c 0.12, \mathrm{MeOH})$. The molecular formula of $\mathbf{1}$ was established as $\mathrm{C}_{20} \mathrm{H}_{34} \mathrm{O}_{3}$ from a protonated molecule at $\mathrm{m} / z$ 323.2574 $[\mathrm{M}+\mathrm{H}]^{+}$(calculated for $\mathrm{C}_{20} \mathrm{H}_{35} \mathrm{O}_{3}, 323.2581$ ) in the HRESIMS spectrum. The cembranoid skeleton of $\mathbf{1}$ was indicated by the characteristic resonances for an isopropyl moiety at $\delta_{\mathrm{H}}$ $1.86(\mathrm{~m}, \mathrm{H}-15), 0.98\left(\mathrm{~d}, J=6.9 \mathrm{~Hz}, \mathrm{H}_{3}-16\right)$ and $0.86(\mathrm{~d}, J=7.2 \mathrm{~Hz}$, $\mathrm{H}_{3}$-17) in the ${ }^{1} \mathrm{H}$ NMR spectrum (Table 1), as well as their corresponding carbon resonances at $\delta_{\mathrm{C}} 35.2$ (C-15), 16.1 (C-16) and 19.1 (C-17) in the ${ }^{13} \mathrm{C}$ NMR spectrum (Table 2) (Wang, et al., 2020). Compound 1 showed superimposable ${ }^{1} \mathrm{H}$ and ${ }^{13} \mathrm{C}$ NMR resonances as the known compound $\mathbf{9}$, but lacked resonances for an acetyl moiety. In the ${ }^{13} \mathrm{C}$ NMR spectrum of 1 , two oxygenated tertiary carbons resonances at $\delta_{\mathrm{C}} 88.5(\mathrm{C}-1)$ and $84.1(\mathrm{C}-12)$ were assignable to a tetrahydrofuran structure formed by cyclization between $\mathrm{C}-1$ and $\mathrm{C}-12$ through an ether bond, which was indicated by the $\mathrm{HMBC}$ correlations between $\mathrm{H}-15 / \mathrm{C}-14, \mathrm{H}$ 15/C-1, $\mathrm{H}_{3}-20 / \mathrm{C}-12$ and $\mathrm{H}_{3}-20 / \mathrm{C}-13$ (Figure 2) (Wang J. J. et al., 


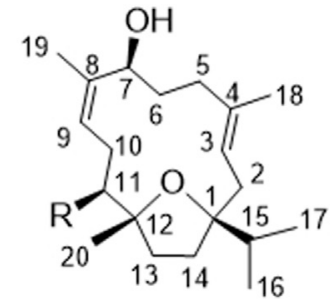

1: $\mathrm{R}=\mathrm{OH}$

9: $\mathrm{R}=\mathrm{OAC}$

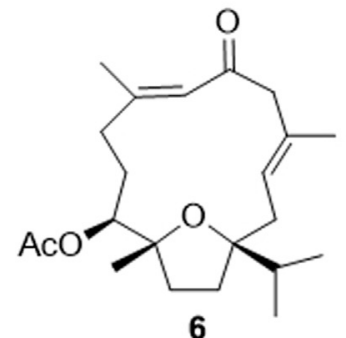<smiles>[R]C1C/C=C(/C)[C@@H](O)CC/C(C)=C/C[C@@]2(C(C)C)CC[C@@](C)(C[C@@H]1C)O2</smiles>

2: $\mathrm{R}=\mathrm{OH}$

3: $\mathrm{R}=\mathrm{OAC}$

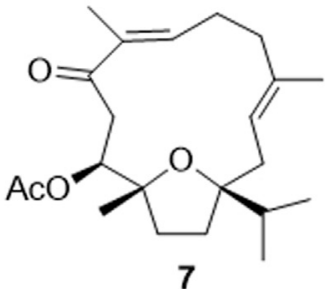<smiles>[R]C(CCC(C)=CCCC(C)C(=O)CC1(C(C)C)CCC1(C)C(C)C)C(C)C</smiles>

4: $\mathrm{R}=\mathrm{OH}$

5: $\mathrm{R}=\mathrm{OAC}$

FIGURE 1 | Chemical structures of compounds 1-9.

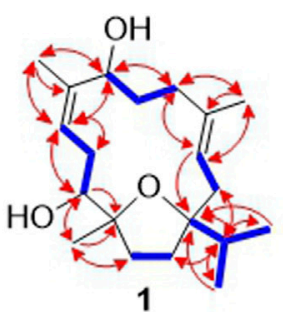

1

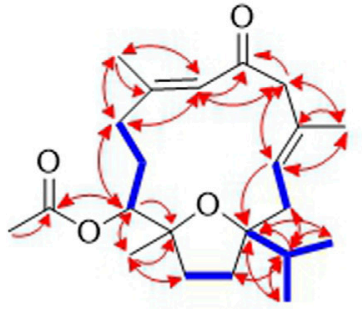

6

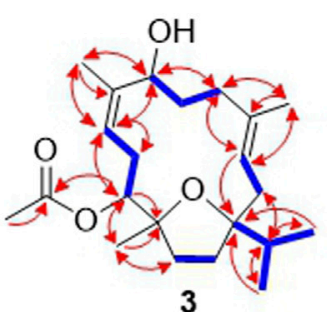

3

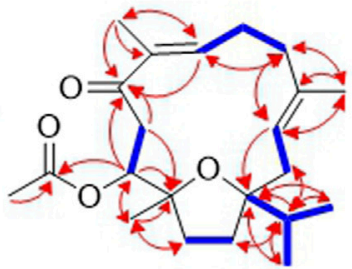

7

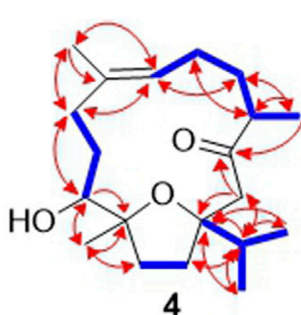

4

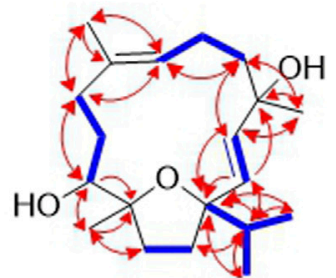

8

\section{${ }^{1} \mathrm{H}-{ }^{1} \mathrm{H} \cos P \overparen{\mathrm{HMBC}}$}

FIGURE 2 | Key ${ }^{1} \mathrm{H}-{ }^{1} \mathrm{H}$ COSY and HMBC correlations of compounds 1, 3-4, 6-8.

2019; Wang Y. G. et al., 2019; Wang et al., 2020). Further functionalities were suggested to be two tri substituted olefins $\left[\delta_{\mathrm{H}} 5.27(\mathrm{H}-3), \delta_{\mathrm{C}} 121.9(\mathrm{C}-3), 130.7(\mathrm{C}-4) ; \delta_{\mathrm{H}} 5.34(\mathrm{H}-9), \delta_{\mathrm{C}}\right.$ $121.9(\mathrm{C}-9), 140.7(\mathrm{C}-8)]$ and two oxygenated methines $\left[\delta_{\mathrm{H}} 4.63\right.$ $(\mathrm{H}-7), \delta_{\mathrm{C}} 68.3(\mathrm{C}-7) ; \delta_{\mathrm{H}} 3.45(\mathrm{H}-11), \delta_{\mathrm{C}} 76.9$ (C-11)]. The assignment of a $\Delta^{3,4}$ olefin moiety in 1 was accomplished by the HMBC correlations between $\mathrm{H}-3 / \mathrm{C}-1, \mathrm{H}-3 / \mathrm{C}-18$, and $\mathrm{H}_{3}-18$ / $\mathrm{C}-3$, while a hydroxy group at $\mathrm{C}-11$ was evident by the $\mathrm{HMBC}$ correlations between $\mathrm{H}-11 / \mathrm{C}-20$ and $\mathrm{H}_{3}-20 / \mathrm{C}-11$. On the other hand, the $\Delta^{8,9}$ olefin and the second hydroxy group at $\mathrm{C}-7$ were evident by the HMBC correlations between H-11/C-9, H-9/C-11, $\mathrm{H}_{3}-19 / \mathrm{C}-9, \mathrm{H}_{3}-19 / \mathrm{C}-7, \mathrm{H}-7 / \mathrm{C}-19$, and H-9/C-19. Thus, the planar structure of compound 1 was concluded as 1:12-epoxycembra-3,8-dien-7,11-diol.

The relative configurations were elucidated by interpretation of the NOESY data (Figure 3). The cis relationship between C-1 isopropyl 


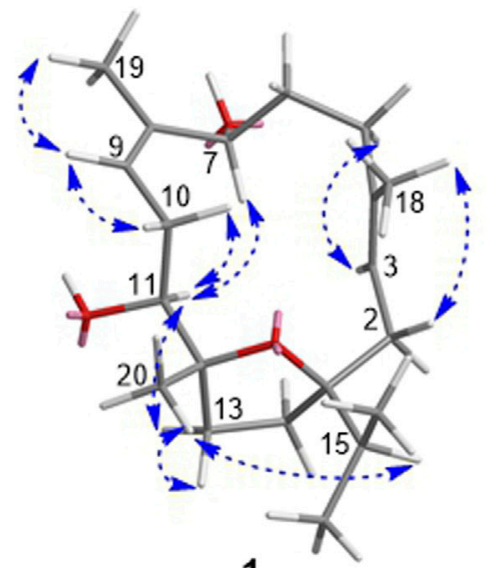

1

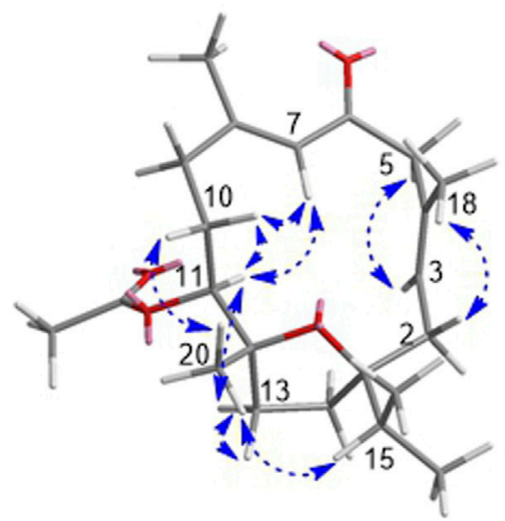

6

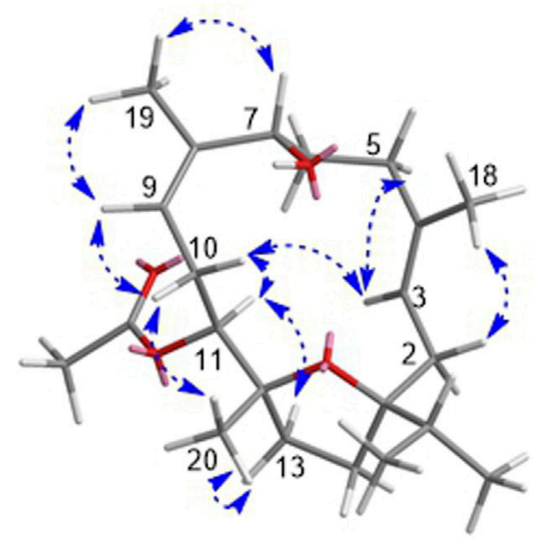

3

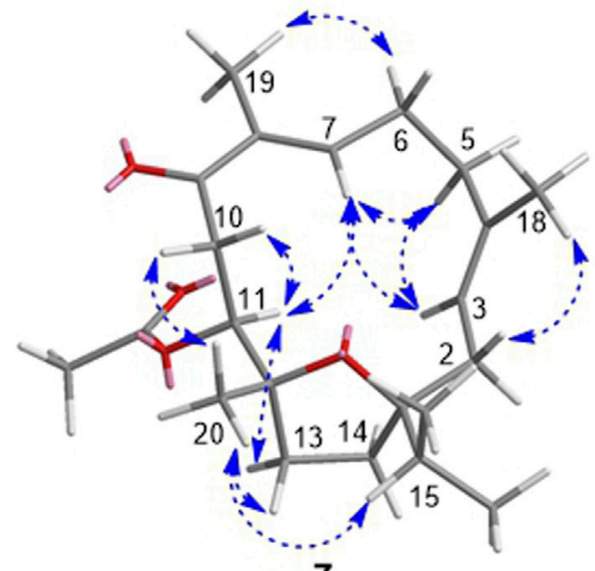

7

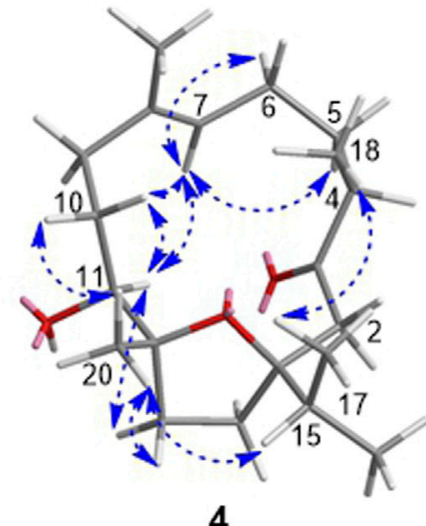

4

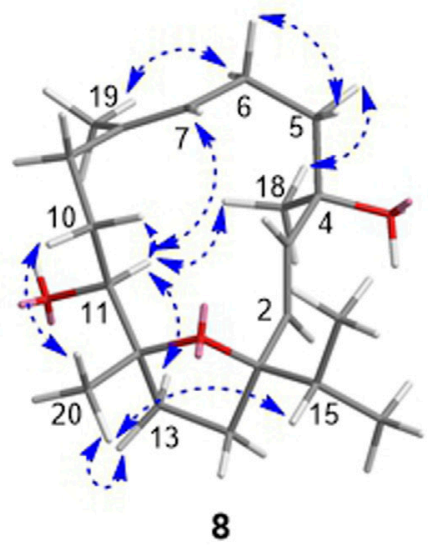

FIGURE $\mathbf{3}$ | Key NOESY correlations of compounds 1, 3-4, 6-8.

moiety and C-12 methyl moiety was evident by the NOESY correlation between $\mathrm{H}-15 / \mathrm{H}_{3}-20$. The $\beta$-orientation of $\mathrm{OH}-11$ was deduced by the NOESY correlations between $\mathrm{H}_{3}-20 / \mathrm{H}-13 \beta$, and $\mathrm{H}-$ $11 / \mathrm{H}-13 \alpha$, while the $\beta$-orientation of $\mathrm{OH}-7$ was deduced by the NOESY correlation between $\mathrm{H}-7 / \mathrm{H}-11$. The $3 E, 8 Z$ configurations of the olefinic geometries were determined by NOESY correlations between $\mathrm{H}-3 / \mathrm{H}-5 \alpha, \mathrm{H}_{3}-18 / \mathrm{H}-2 \beta$ and $\mathrm{H}-9 / \mathrm{H}_{3}-19$. Finally, the structure of $\mathbf{1}$ including the absolute configurations was determined by singlecrystal X-ray diffraction analysis using $\mathrm{Cu} \mathrm{K} \alpha$ radiation [CDCC number, 2035706, Flack parameter $=-0.2$ (2)] (Figure 4). Thus, the structure of sacraoxide A (1) was unambiguously determined as (1S,7S,11S,12R)-1:12-epoxy-cembra-3E,8Z-dien-7,11-diol.

Sacraoxide B (3) was isolated as colorless oil $[\alpha]_{\mathrm{D}}^{25}-4.1(c 0.10$, $\mathrm{MeOH})$. The molecular formula of 3 was established as $\mathrm{C}_{22} \mathrm{H}_{36} \mathrm{O}_{4}$ from the HRESIMS positive-ion at $\mathrm{m} / z$ 387.2513 $[\mathrm{M}+\mathrm{Na}]^{+}$ (calculated for $\mathrm{C}_{22} \mathrm{H}_{36} \mathrm{O}_{4} \mathrm{Na}, 387.2511$ ). The $1 \mathrm{D}$ and 2D NMR spectroscopic data of $\mathbf{3}$ were mostly compatible with those of the known compound 2, except for the downfield shifted $\mathrm{H}-11$ resonance $\left(\delta_{\mathrm{H}} 5.13\right)$ and the existence of a set of resonances for an acetyl group [ $\left.\delta_{\mathrm{H}} 2.03\left(\mathrm{H}_{3}-2^{\prime}\right), \delta_{\mathrm{C}} 21.3\left(\mathrm{C}-2^{\prime}\right), 171.8\left(\mathrm{C}-1^{\prime}\right)\right]$ (Tables 1, 2). The position of the acetyl group at $\mathrm{C}-11$ was further determined by the HMBC correlation between $\mathrm{H}-11 / \mathrm{C}-1^{\prime}$. In the NOESY spectrum, a key correlation was observed between $\mathrm{H}-7 / \mathrm{H}_{3}-19$ but not of $\mathrm{H}-7 / \mathrm{H}-$ 11 , indicating that $\mathrm{OH}-7$ is $\alpha$-orientated (Figure 3). Meanwhile, the ECD spectra of $\mathbf{3}$ and $\mathbf{2}$ also revealed high similarities, suggesting the same absolute configurations.

In order to determine the absolute configurations of compound 3, the modified Mosher's method was carried out (Li et al., 2007). Compound 2 was separately esterified with $(S)$ and $(R)$-MTPA chloride to give corresponding $(R)$ and $(S)$ MTPA esters, $\mathbf{2} \mathbf{a}$ and $\mathbf{2 b}$, respectively. As a result, only monosubstituted $(R / S)$-MTPA esters were obtained as the products, which were demonstrated by the HRESIMS and NMR spectroscopic data. The fact that $\mathrm{OH}-7$ was esterified, was indicated by the downfield shifted $\mathrm{H}-7$ resonances and the HMBC correlations between H-7/MTPA-C- $1^{\prime}$ in $\mathbf{2 a}$ and $\mathbf{2 b}$. The regioselectivity was in accordance with cis-relationship between $\mathrm{CH}_{3}-20$ and $\mathrm{OH}-11$ causing steric hindrance effect. The distribution of $\Delta \delta$ values between $\mathbf{2 b}$ and $\mathbf{2 a}$ indicated the $7 R$-configuration of $\mathbf{2}$ (Figure 5). Thus, the structure of sacraoxide B (3) was determined as $(1 S, 7 R, 11 S, 12 R)-1: 12$ epoxy-11-acetoxy-cembra-3E,8Z-dien-7-ol.

Sacraoxide C (4) was isolated as colorless oil $[\alpha]_{\mathrm{D}}^{25}+10.4(c$ $0.10, \mathrm{MeOH})$. The molecular formula of 4 was established as 


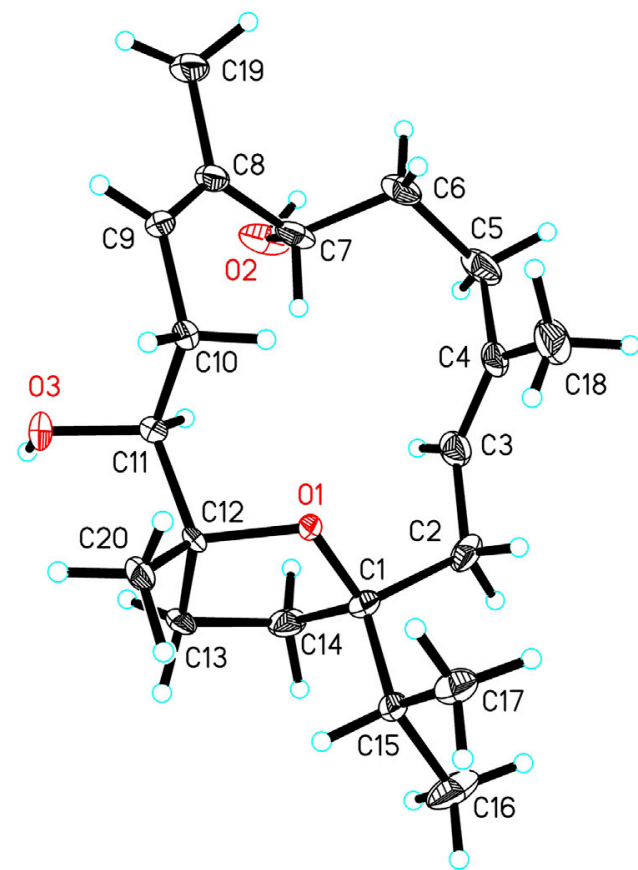

FIGURE 4 | X-ray ORTEP drawing of compound $\mathbf{1 .}$

$\mathrm{C}_{20} \mathrm{H}_{34} \mathrm{O}_{3}$ from a protonated molecule at $\mathrm{m} / z$ 323.2581 $[\mathrm{M}+\mathrm{H}]^{+}$ (calculated for $\mathrm{C}_{20} \mathrm{H}_{35} \mathrm{O}_{3}, 323.2586$ ) in the HRESIMS spectrum. Compound 4 is a cembranoid possessing a tetrahydrofuran ring and 11-hydroxy group, which was deduced by the same NMR spectroscopic data analysis process as aforementioned in compound 1. However, in the ${ }^{1} \mathrm{H}$ and ${ }^{13} \mathrm{C}$ NMR spectra of 4 , the resonances of a methyl doublet at $\delta_{\mathrm{H}} 1.04\left(\mathrm{~d}, J=7.2 \mathrm{~Hz}, \mathrm{H}_{3^{-}}\right.$ 18 ), and a carbonyl group at $\delta_{\mathrm{C}} 213.4$ (C-3) were observed (Tables 1, 2). Their positions were assigned by the $\mathrm{HMBC}$ correlations between H-15/C-2, $\mathrm{H}_{2}-2 / \mathrm{C}-3, \quad \mathrm{H}_{3}-18 / \mathrm{C}-3$ (Figure 2). Meanwhile, the position of the trisubstituted olefin was determined at $\Delta^{7,8}$ by the HMBC correlations between $\mathrm{H}_{3}-19$ / $\mathrm{C}-7, \mathrm{H}-7 / \mathrm{C}-5$, and $\mathrm{H}_{3}-18 / \mathrm{C}-5$, as well as the ${ }^{1} \mathrm{H}_{-}{ }^{1} \mathrm{H}$ COSY correlations between $\mathrm{H}_{3}-18 / \mathrm{H}-4, \mathrm{H}-4 / \mathrm{H}-5, \mathrm{H}-5 / \mathrm{H}-6$, and $\mathrm{H}-6 /$ $\mathrm{H}-7$. The trans-olefinic geometry of $\Delta^{7,8}$ was determined by the NOESY correlations between $\mathrm{H}-7 / \mathrm{H}-10 \alpha$, and the absence of $\mathrm{H}-$ $7 / \mathrm{H}_{3}-19$ (Figure 3). Furthermore, $\mathrm{OH}-11$ and $\mathrm{CH}_{3}-18$ were both $\beta$-orientated, which were deduced by the NOESY correlations between $\mathrm{H}_{3}-20 / \mathrm{H}-13 \beta$, H-11/H-13 $\alpha$, and $\mathrm{H}_{3}-17 / \mathrm{H}_{3}-18$.

The absolute configurations of $\mathbf{4}$ were determined by comparison of experimental and calculated ECD spectra. Time dependent density functional theory (TDDFT) at the B3LYP/6-311++G $(2 \mathrm{~d}, p)$ level with IEFPCM in $\mathrm{MeOH}$ was used to calculate the ECD spectra of two enantiomers of 4 . The experimental ECD spectrum of 4 showed a positive cotton effect $(\mathrm{CE})$ at $292 \mathrm{~nm}\left(\mathrm{n} \rightarrow \pi^{*}\right)$, a negative CE at $239 \mathrm{~nm}(\pi \rightarrow$ $\left.\pi^{\star}\right)$, and a positive CE at $205 \mathrm{~nm}\left(\mathrm{n} \rightarrow \sigma^{\star}\right)$, which coincided well with the calculated ECD spectrum of $(1 S, 4 R, 7 E, 11 S, 12 R)$ 4 (Figure 6), Thus, the structure of sacraoxide $C$ (4) was determined as $(1 S, 4 R, 11 S, 12 R)$-1:12-epoxy-11-hydroxycembra-7E-en-3-one.

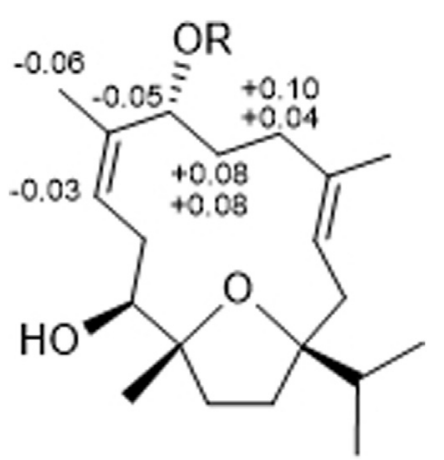

$$
\begin{aligned}
& \text { 2a } \mathrm{R}=(R) \text {-MTPA } \\
& \text { 2b } \mathrm{R}=(S) \text {-MTPA }
\end{aligned}
$$

FIGURE $\mathbf{5}$ | Results of compound $\mathbf{2}$ with the modified mosher's method $\left(\Delta \delta_{H}=\delta_{S}-\delta_{R}\right)$

Sacraoxide D (5) is an acetylated derivate of compound 4, which was indicated by both HRESIMS and NMR spectroscopic data. The acetoxy group was determined at $\mathrm{C}-11$, since the proton resonance of $\mathrm{H}-11\left(\delta_{\mathrm{H}} 5.13\right)$ was observed downfield shifted (Table 1), and the HMBC correlation between $\mathrm{H}-11 / \mathrm{C}-1^{\prime}$ was also observed. The ECD spectra of $\mathbf{5}$ and $\mathbf{4}$ revealed high similarities, suggesting the same absolute configurations. Thus, the structure of sacraoxide D (5) was determined as $(1 S, 4 R, 11 S, 12 R)$-1:12-epoxy-11-acetoxy-cembra-7E-en-3-one.

Sacraoxide E (6) was isolated as colorless oil $[\alpha]_{\mathrm{D}}^{25}-64.0(c$ $0.10, \mathrm{MeOH})$. The molecular formula of 6 was established as $\mathrm{C}_{22} \mathrm{H}_{34} \mathrm{O}_{4}$ from the HRESIMS positive-ion at $m / z$ 385.2354 [M + $\mathrm{Na}]^{+}$(calculated for $\mathrm{C}_{22} \mathrm{H}_{34} \mathrm{O}_{4} \mathrm{Na}, 385.2355$ ). Compound 6 is also a cembranoid, with a tetrahydrofuran ring and $11 \beta$-acetoxy group, which was suggested by its superimposable resonances in comparison with structurally similar compounds $\mathbf{9 , 3}$, and $\mathbf{5}$. Other functionalities were suggested to be two trisubstituted olefin groups and a carbonyl moiety. One olefin was assigned to be a trans $-\Delta^{3,4}$ moiety by the HMBC correlations between $\mathrm{H}-3$ / $\mathrm{C}-1, \mathrm{H}-3 / \mathrm{C}-18$, and $\mathrm{H}_{3}-18 / \mathrm{C}-3$, as well as the NOESY correlations between $\mathrm{H}-3 / \mathrm{H}-5 \alpha$, and $\mathrm{H}-2 \beta / \mathrm{H}-18$ (Figures 2, 3). In addition, the presence of an $\alpha, \beta$-unsaturated ketone moiety was suggested by observation of a downfield olefinic proton at $\delta_{\mathrm{H}} 6.53(\mathrm{H}-7)$ and a carbonyl carbon resonance at $\delta_{\mathrm{C}} 201.2$ (C-6), which were assigned as 7,8-en-6-one by the ${ }^{3} \mathrm{~J}$-HMBC correlations between $\mathrm{H}_{3}-19 / \mathrm{C}-7, \mathrm{H}-7 / \mathrm{C}-5$, and $\mathrm{H}_{3}-18 / \mathrm{C}-5$, as well as the ${ }^{2} \mathrm{~J}-\mathrm{HMBC}$ correlations between $\mathrm{H}-5 / \mathrm{C}-6$, and $\mathrm{H}-7 / \mathrm{C}-6$. The trans-olefinic geometry of $\Delta^{7,8}$ was evident by the NOESY correlation between $\mathrm{H}-10 \alpha / \mathrm{H}-7$. The experimental ECD spectrum of 6 showed a negative CE at $348 \mathrm{~nm}\left(\mathrm{n} \rightarrow \pi^{*}\right)$, a positive CE at $280 \mathrm{~nm}(\pi \rightarrow$ $\left.\pi^{*}\right)$, a negative $\mathrm{CE}$ at $241 \mathrm{~nm}\left(\pi \rightarrow \pi^{*}\right)$, and a positive CE at $203 \mathrm{~nm}\left(\mathrm{n} \rightarrow \sigma^{\star}\right)$, which coincided well with the calculated ECD spectrum of $(1 S, 3 E, 7 E, 11 S, 12 R)-6$ (Figure 7). Thus, the structure of sacraoxide $\mathrm{E}(6)$ was determined as $(1 S, 11 S, 12 R)$-1:12-epoxy11-acetoxy-cembra-3E,7E-dien-6-one.

Sacraoxide F (7) has the same molecular formula of $\mathrm{C}_{22} \mathrm{H}_{34} \mathrm{O}_{4}$ as 6, which was established from a protonated molecule at $\mathrm{m} / \mathrm{z}$ 


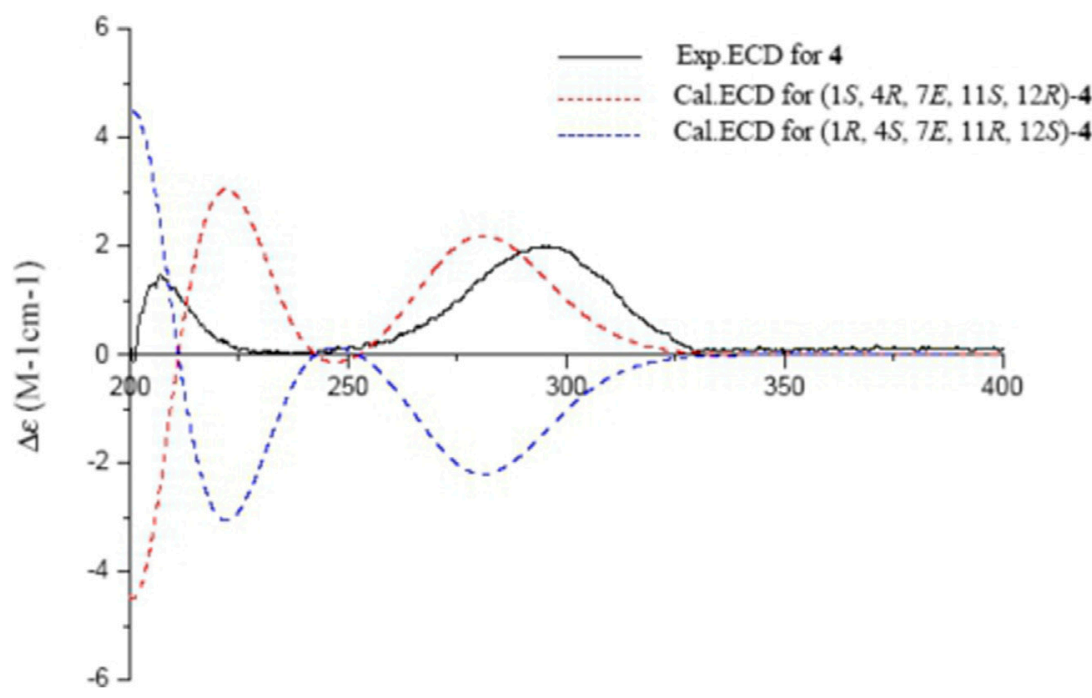

FIGURE 6 | Experimental and calculated ECD spectra of 4 and its enantiomer (red and blue calculated at the B3LYP/6-311++G (2d,p)//B3LYP/6-31+G (d,p) level in $\mathrm{CH}_{3} \mathrm{OH}$; black, experimental in $\mathrm{CH}_{3} \mathrm{OH}$ ).

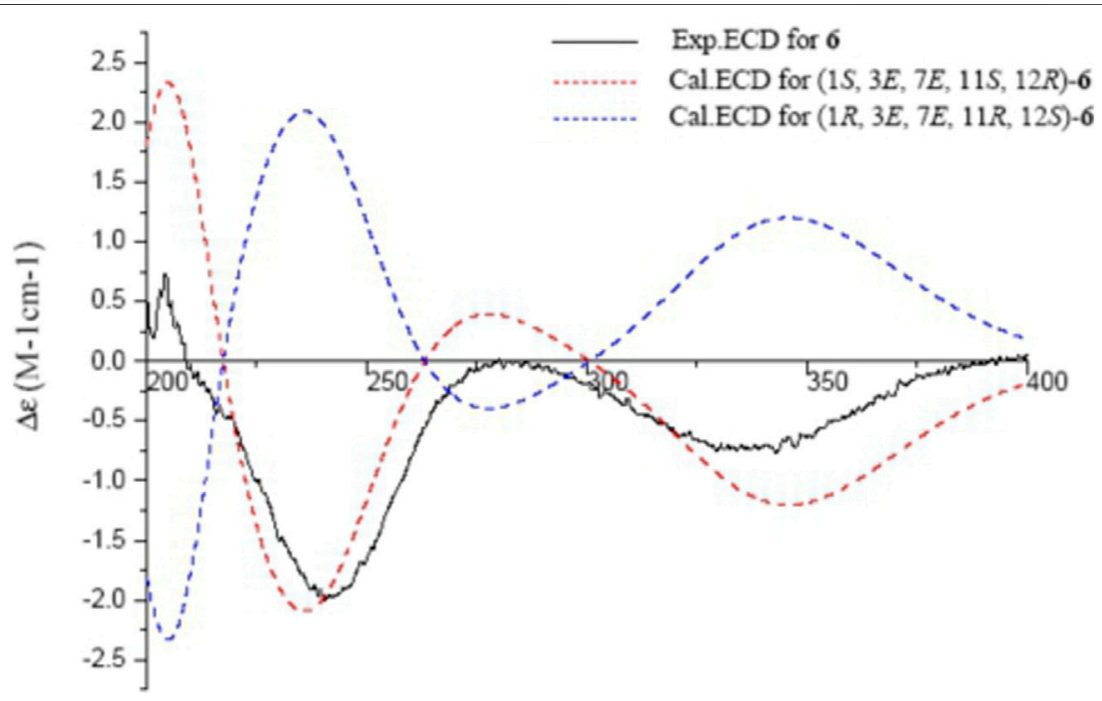

FIGURE 7 | Experimental and calculated ECD spectra of 6 and its enantiomer (red and blue calculated at the B3LYP/6-311++G (2d,p)//B3LYP/6-31+G (d,p) level in $\mathrm{CH}_{3} \mathrm{OH}$; black, experimental in $\mathrm{CH}_{3} \mathrm{OH}$ ).

363.2504 $[\mathrm{M}+\mathrm{H}]^{+}$(calculated for $\mathrm{C}_{22} \mathrm{H}_{35} \mathrm{O}_{4}, 363.2535$ ) in the HRESIMS spectrum. The functionalities of the cembranoid skeleton of compound 7 included a tetrahydrofuran ring and $11 \beta$-acetoxy group, as well as two trisubstituted olefin groups and a carbonyl moiety. Detailed analyses of the 2D NMR spectroscopic data assigned the $\alpha, \beta$-unsaturated ketone moiety as 7,8-en-9-one by the HMBC correlations between $\mathrm{H}_{3}-19 / \mathrm{C}-7$, $\mathrm{H}_{3}-19 / \mathrm{C}-9$, and H-11/C-9 (Figure 2). Additionally, the experimental ECD spectrum of 7 coincided well with calculated ECD spectrum of $(1 S, 3 E, 7 E, 11 S, 12 R)-7$ (Figure 8). Thus, the structure of sacraoxide F (7) was determined as
$(1 S, 11 S, 12 R)$-1:12-epoxy-11 $\beta$-acetoxy-cembra-3E,7E-dien9-one.

Sacraoxide $\mathrm{G}(\mathbf{8})$ was isolated as white amorphous powder $[\alpha]$ ${ }_{\mathrm{D}}^{25}+115.0$ (c 0.11, MeOH). The molecular formula of 8 was established as $\mathrm{C}_{20} \mathrm{H}_{34} \mathrm{O}_{3}$ from the HRESIMS positive-ion at $\mathrm{m} / z$ $305.2583\left[\mathrm{M}-\mathrm{H}_{2} \mathrm{O}+\mathrm{H}\right]^{+}$(calculated for $\mathrm{C}_{20} \mathrm{H}_{33} \mathrm{O}_{2}, 305.2475$ ). Compound 8 is a cembranoid with a tetrahydrofuran ring and $\mathrm{OH}-11 \beta$ group, which was suggested by its superimposable resonances in comparison with structurally similar compounds $\mathbf{1}, 2$ and 4 . In comparison to compound $\mathbf{4}$, the different functionalities of $\mathbf{8}$ were suggested to be a disubstituted trans- 


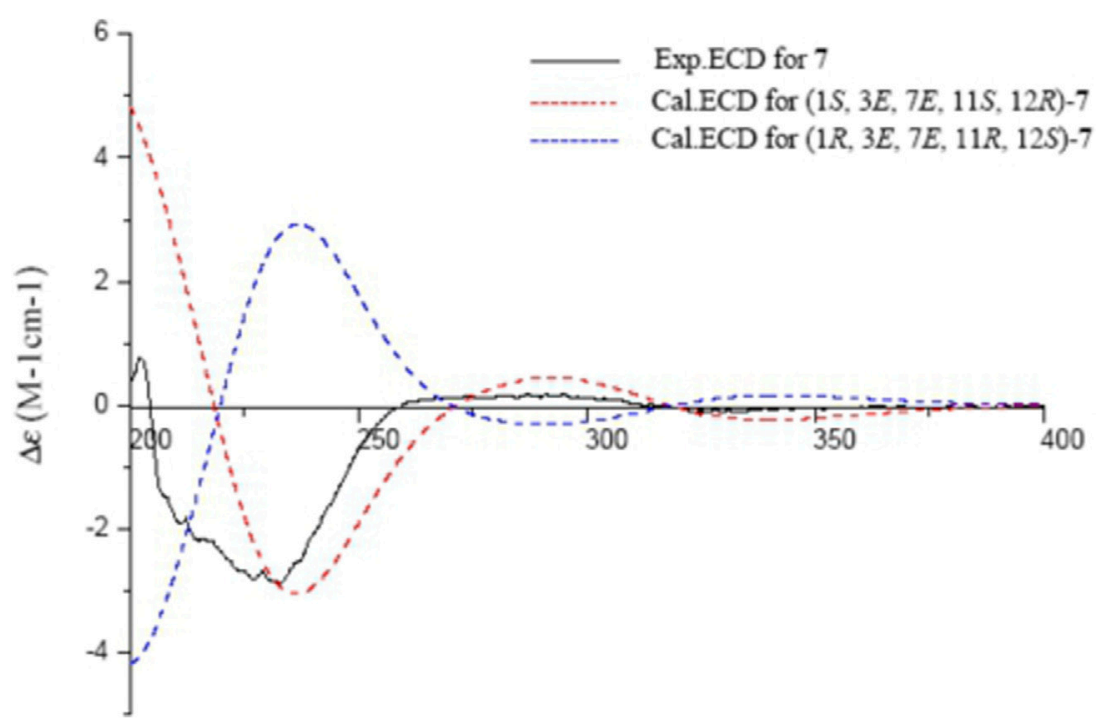

FIGURE 8| Experimental and calculated ECD spectra of 7 and its enantiomer (red and blue calculated at the B3LYP/6-311++G (2d,p)//B3LYP/6-31+G (d,p) level in $\mathrm{CH}_{3} \mathrm{OH}$; black, experimental in $\mathrm{CH}_{3} \mathrm{OH}$ ).

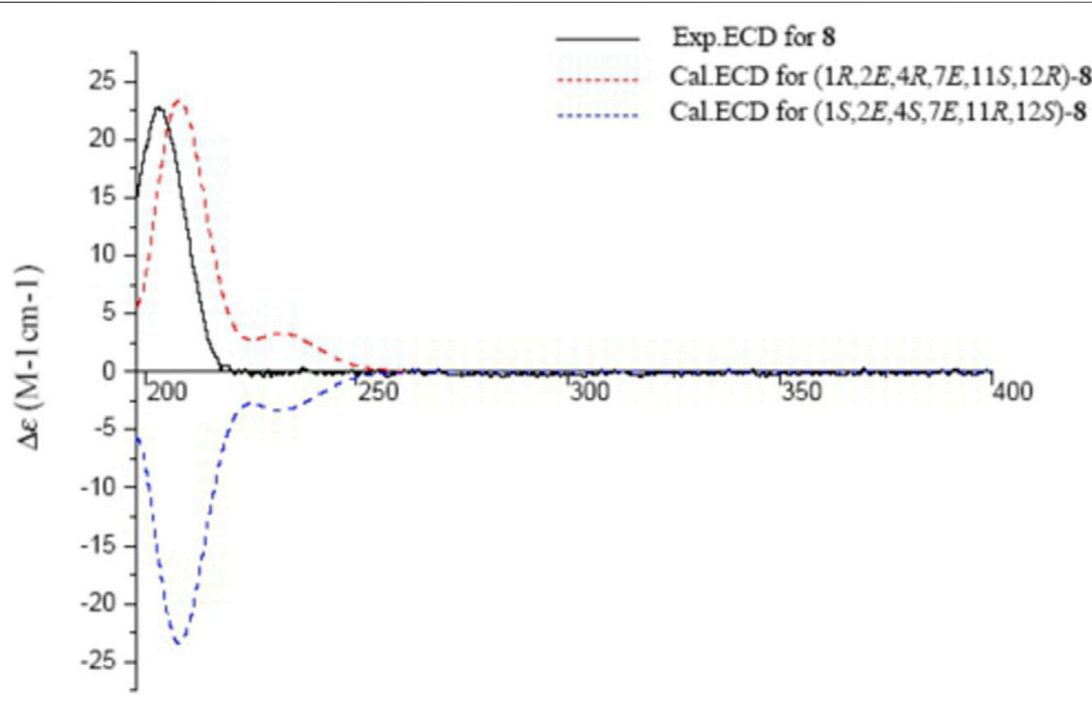

FIGURE 9 | Experimental and calculated ECD spectra of 8 and its enantiomer (red and blue calculated at the B3LYP/6-311++G (2d,p)//B3LYP/6-31+G (d,p) level in $\mathrm{CH}_{3} \mathrm{OH}$; black, experimental in $\mathrm{CH}_{3} \mathrm{OH}$ ).

olefin $\left[\delta_{\mathrm{H}} 5.54(\mathrm{~d}, J=15.4 \mathrm{~Hz}, \mathrm{H}-2), 5.81(\mathrm{~d}, J=15.4 \mathrm{~Hz}, \mathrm{H}-3)\right]$, and an oxygenated quaternary carbon $\left[\delta_{\mathrm{C}} 74.4(\mathrm{C}-4)\right]$ (Tables 2, 3). Their positions were assigned by the $\mathrm{HMBC}$ correlations between $\mathrm{H}-3 / \mathrm{C}-1, \mathrm{H}-2 / \mathrm{C}-15, \mathrm{H}_{3}-18 / \mathrm{C}-3$, and H-2/C-4 (Figure 2). The $\beta$-orientation of $\mathrm{OH}-4$ and $\mathrm{OH}-11$ were evident by the NOESY correlations between $\mathrm{H}_{3}-20 / \mathrm{H}-13 \beta, \mathrm{H}-13 \alpha / \mathrm{H}-11$ and $\mathrm{H}_{3}-18 / \mathrm{H}-11$ (Figure 3). The experimental ECD spectrum of 8 coincided well with the calculated ECD spectrum of $(1 R, 2 E, 4 R, 7 E, 11 S, 12 R)-\mathbf{8}$ (Figure 9). Thus, the structure of sacraoxide $G(8)$ was determined as $(1 R, 4 R, 11 S, 12 R)-1: 12$ epoxy-cembra-2E,7E-dien-4,11-diol.
Nitric oxide (NO) plays significant roles in immune and inflammatory responses. The inhibition of NO release may be considered therapeutic in the treatment of inflammatory diseases (Strowig et al., 2012). Taking account of the traditional usage of the olibanum, cembranoids (1-9) were evaluated for their inhibitory activities against lipopolysaccharide (LPS)-induced NO production in RAW 264.7 mouse monocyte-macrophages. Compounds 6 and 7 showed the most potent inhibitory activities with the $\mathrm{IC}_{50}$ values of 36.4 and $24.9 \mu \mathrm{M}$ respectively, while the others were less active or inactive (Table 4). In addition, the MTT assay indicated that none of these compounds showed cytotoxicity in RAW264.7 cells at a 
TABLE 4 | Inhibitory activity of compounds 1-9 against NO production in RAW 264.7 cells

\begin{tabular}{lc} 
Compound $^{\mathbf{a}}$ & $\mathbf{I C}_{\mathbf{5 0}}(\boldsymbol{\mu} \mathbf{M})$ \\
\hline $\mathbf{4}$ & $72.1 \pm 5.1$ \\
$\mathbf{6}$ & $36.4 \pm 2.9$ \\
$\mathbf{7}$ & $24.9 \pm 1.7$ \\
HSS $^{\mathrm{b}}$ & $52.2 \pm 4.5$ \\
Indomethacin $^{\mathrm{b}}$ & $11.9 \pm 0.6$
\end{tabular}

${ }^{a}$ Compounds 1-3, 5, and 8-9 showed activities with $I C_{50}>100 \mu \mathrm{M}$.

${ }^{b}$ HSS, (hydrocortisone sodium succinate) and indomethacin were used as a positive control. Data are presented based on three experiments.

concentration of $50 \mu \mathrm{M}$. The presence of an $\alpha, \beta$-unsaturated carbonyl functionality seems essential for the inhibitory activity.

\section{CONCLUSION}

A phytochemical investigation on the gum resin of $B$. sacra resulted in the isolation and structural elucidation of seven undescribed and two known cembranoids. These cembranoids possess a common tertrahydrofuran ring structure through an ether bond between $\mathrm{C}-1$ and C-12. The structures including absolute configurations were determined by extensive physicochemical and spectroscopic analysis, as well as ECD calculation, modified Mosher's method and X-ray diffraction crystallography. Compounds 4, 6, and 7 displayed inhibitory activities against LPS-induced NO production in RAW 264.7 cells with $\mathrm{IC}_{50}$ values ranging from 24.9 to $72.1 \mu \mathrm{M}$. These findings will be of particular value for further studies of structurally interesting cembranoids with biological activities from the genus of Boswellia.

\section{REFERENCES}

Al-Harrasi, A., Csuk, R., Khan, A., and Hussain, J. (2019). Distribution of the antiinflammatory and anti-depressant compounds: incensole and incensole acetate in genus Boswellia. Phytochem. 161, 28-40. doi:10.1016/j.phytochem.2019. 01.007

Banno, N., Akihisa, T., Yasukawa, K., Tokuda, H., Tabata, K., Nakamura, Y., et al. (2006). Anti-inflammatory activities of the triterpene acids from the resin of Boswellia carteri. J. Ethnopharmacol. 107, 249-253. doi:10.1016/j.jep.2006. 03.006

Jin, X., Song, S. Q., Wang, J., Zhang, Q. Z., Qiu, F., and Zhao, F. (2016). Tiliroside, the major component of Agrimonia pilosa Ledeb ethanol extract, inhibits MAPK/JNK/p38-mediated inflammation in lipopolysaccharide-activated RAW 264.7 macrophages. Exp. Ther. Med. 12, 499-505. doi:10.3892/etm. 2016.3305

Li, W., Wei, K., Fu, H., and Koike, K. (2007). Structure and absolute configuration of clerodane diterpene glycosides and a rearranged cadinane sesquiterpene glycoside from the stems of Tinospora sinensis. J. Nat. Prod. 70, 1971-1976. doi:10.1246/bcsj.71.273

Li, Y., and Pattenden, G. (2011). Perspectives on the structural and biosynthetic interrelationships between oxygenated furanocembranoids and their polycyclic congeners found in corals. Nat. Prod. Rep. 28, 1269-1310. doi:10.1039/ C1NP00023C

Moussaieff, A., Gross, M., Nesher, E., Tikhonov, T., Yadid, G., and Pinhasov, A. (2012). Incensole acetate reduces depressive-like behavior and modulates

\section{DATA AVAILABILITY STATEMENT}

The datasets presented in this study can be found in online repositories. The names of the repository/repositories and accession number(s) can be found in the article/ Supplementary Material.

\section{AUTHOR CONTRIBUTIONS}

$\mathrm{BZ}, \mathrm{DL}$, and $\mathrm{WJ}$ were responsible for the isolation of compounds. $\mathrm{BZ}$ and $\mathrm{KO}$ elucidated the structures. FZ and $\mathrm{KH}$ tested NO inhibitory effects of the compounds. $\mathrm{BZ}$ and WL interpreted the data, and wrote the paper. WL, KK, and FQ revised the manuscript. FQ was the project leaders organizing and guiding the experiment. All authors read and approved the final manuscript.

\section{FUNDING}

This research was supported financially by the National Key Research and Development Program of China (2019YFC1711000), and the High-end Manufacturing Innovation-driven Development Project of Xiqing District, Tianjin (cgzh-cgk-2018).

\section{SUPPLEMENTARY MATERIAL}

The Supplementary Material for this article can be found online at: https://www.frontiersin.org/articles/10.3389/fchem.2021.649287/ full\#supplementary-material.

hippocampal BDNF and CRF expression of submissive animals. J. Psychopharmacol. (Oxford) 26, 1584-1593. doi:10.1177/0269881112458729

Pollastro, F., Golin, S., Chianese, G., Putra, M. Y., Moriello, A. S., and Petrocellis, L. D. (2016). Neuroactive and anti-inflammatory Frankincense cembranes: a structureactivity study. J. Nat. Prod. 79, 1762-1768. doi:10.1021/acs.jnatprod.6b00141

Strowig, T., Henao-Mejia, J., Elinav, E., and Flavell, R. (2012). Inflammasomes in health and disease. Nature 481, 278-286. doi:10.1038/nature10759

Takada, Y., Ichikawa, H., Badmaev, V., and Aggrwal, B. B. (2006). Acetyl-11-Keto$\beta$-Boswellic acid potentiates apoptosis, inhibits invasion, and abolishes osteoclastogenesis by suppressing NF- $\mathrm{BB}$ and NF- $\mathrm{BB}$-Regulated gene expression. J. Immunol. 176, 3127-3140. doi:10.4049/jimmunol.176.5.3127

Wahlberg, I., Eklund, A. M., Ab, R., and Sweden, S. (1992). Cembranoids, pseudopteranoids, and cubitanoids of natural occurrence. Fortschritte Der Chem. Organischer Naturstoffe. 59, 141-294. doi:10.1007/978-3-7091-9150-7_2

Wang, J. J., Sun, H. R., Suo, X. Y., Wang, X., and Ji, T. F. (2020). Ten undescribed cembrane-type diterpenoids from the gum resin of Boswellia sacra and their biological activities. Phytochemistry 177, 112425. doi:10.1016/j.phytochem. 2020.112425

Wang, J. J., Zhen, B., Hu, J. W., Shi, M. J., Wei, C. J., and Wang, X. (2019). Boscartins L-O: cembrane-type diterpenoids from the gum resin of Boswellia sacra Flueck. Phytochemistry 163, 126-131. doi:10.1016/j.phytochem.2019.03.005

Wang, Y. G., Ren, J., Ma, J., Yang, J. B., Ji, T. F., and Wang, A. G. (2019). Bioactive cembrane-type diterpenoids from the gum-resin of Boswellia carterii. Fitoterapia 137, 104263. doi:10.1016/j.fitote.2019.104263

Xia, G. Y., Yao, T., Zhang, B. Y., Li, Y., Kang, N., and Cao, S. J. (2017). Withapubesides A-D: natural inducible nitric oxide synthase (iNOS) 
inhibitors from Physalis pubescens. Org. Biomol. Chem. 15, 10016-10023. doi:10.1039/С7ОB02551C

Yan, N., Du, Y. M., Zhang, H. B., Liu, Y. H., Zhang, P., and Gong, D. P. (2016). Chemical structures, biosynthesis, bioactivities, biocatalysis and semisynthesis of tobacco cembranoids: an overview. Ind. Crop Prod. 83, 66-80. doi:10.1016/j.indcrop.2015.12.031

Yang, B., Zhou, X. F., Lin, X. P., Liu, J., Peng, Y., and Yang, X. W. (2012). Cembrane diterpenes chemistry and biological properties. Cur. Org. Chem. 16, 1512-1539. doi:10.2174/138527212800672583

Zhang, N., Wei, S. X., Cao, S. J., Zhang, Q., Kang, N., and Ding, L. Q. (2020). Bioactive triterpenoid saponins from the seeds of Aesculus chinensis Bge. var. Chekiangensis. Front. Chem. 7, 908. doi:10.3389/fchem.2019.00908
Conflict of Interest: The authors declare that the research was conducted in the absence of any commercial or financial relationships that could be construed as a potential conflict of interest.

Copyright (๑) 2021 Zhang, Liu, Ji, Otsuki, Higai, Zhao, Li, Koike and Qiu. This is an open-access article distributed under the terms of the Creative Commons Attribution License (CC BY). The use, distribution or reproduction in other forums is permitted, provided the original author(s) and the copyright owner(s) are credited and that the original publication in this journal is cited, in accordance with accepted academic practice. No use, distribution or reproduction is permitted which does not comply with these terms. 